\title{
Nonlinear neutrino oscillations in the expanding Universe
}

\author{
V. Alan Kostelecký and Stuart Samuel* \\ Physics Department, Indiana University, Bloomington, Indiana 47405
}

(Received 2 August 1993)

\begin{abstract}
We derive the equations that govern neutrino and antineutrino behavior in the early Universe. Results are presented from numerical simulations incorporating nonlinear effects of the neutrinoantineutrino background as well as the leading $C P$-symmetric and $C P$-asymmetric terms arising from the interactions with electrons and positrons. A broad spectrum of neutrino and antineutrino behavior is observed, ranging from vacuumlike to smooth. Oscillations exhibiting self-maintained coherence also appear. We identify a variety of neutrino properties arising as a consequence of the nonlinear neutrino self-interactions. The nonlinear effects tend to suppress any $C P$ asymmetry relative to the linear case. An analytical approximation scheme reproducing bulk neutrino behavior is described. We discuss some physical implications of our results.
\end{abstract}

PACS number(s): 95.30.Cq, 12.15.Ff, 14.60.Pq

\section{INTRODUCTION}

In the standard cosmological scenario [1], the early Universe at about a tenth of a second consisted principally of a hot gas of photons, electrons, neutrinos, and their antiparticles in approximate thermodynamic equilibrium at a temperature of about $10 \mathrm{MeV}$. As the Universe expanded and cooled, the neutrino interaction cross sections decreased until, at a temperature of about $2 \mathrm{MeV}$, the neutrino component of the gas decoupled thermally. Subsequently, the electrons and positrons annihilated, thereby reheating the photons relative to the neutrinos. The annihilation also slightly reheated the electron neutrinos relative to the muon or other neutrinos, resulting in an electron-neutrino excess of about $1 \%[2,3]$.

Despite being decoupled from the photons and other leptons, neutrinos influenced nucleosynthesis through neutrino-nucleon interactions until the temperature dropped by about another factor of 2 and attained the "freeze-out" point $T_{f} \approx 0.7 \mathrm{MeV}$ where neutrinos ceased to affect the neutron-proton ratio [4,5]. Subsequently, the primary cosmological role for neutrinos came through their contribution to the energy density of the Universe, which controlled the expansion rate. From the moment of decoupling to the present day, neutrinos evolved according to a Fermi-Dirac distribution, modulo distortions at the $1 \%$ level due to the electron-positron reheating. A Cosmic Background Explorer- (COBE-) type experiment able to detect the cosmic neutrino relic should therefore reveal a Fermi-Dirac distribution with a $1 \%$ distortion at a temperature of about $2 \mathrm{~K}$. Today, there are about $10^{8}$ relic neutrinos per cubic meter.

This canonical picture assumes that the standard electroweak model holds exactly. In particular, neutrinos are taken to be massless, purely left-handed Dirac particles. The known sensitivity of nucleosynthesis to neutrino

\footnotetext{
${ }^{*}$ On leave of absence from the City College of New York.
}

types and properties and the likely sensitivity of other potentially measurable quantities, such as the cosmicneutrino relic [6], dark matter, or large-scale structure, suggests the early Universe can serve as a probe of nonminimal electroweak physics.

In this paper, we consider effects arising in the early Universe from a particular extension of the minimal standard model. We assume the existence of right-handed neutrinos and corresponding neutrino Dirac masses. We suppose that the neutrino masses are small relative to the mean neutrino energy during the relevant time period, so that we work in the neutrino relativistic limit.

New effects can appear in principle as a result of the additional degrees of freedom and also from the possible presence of neutrino oscillations [7]. Since no net flavor change can occur if the numbers of electron and muon neutrinos are equal, oscillations can only play a role in changing the net electron-neutrino excess. Nonetheless, this leaves open the possibility in principle that neutrino oscillations could produce measurable effects on nucleosynthesis or other observable physics [8-12]. A comprehensive analysis of this issue is difficult because the electron-neutrino excess depends on interactions with background fields, including the neutrinos and antineutrinos themselves. As we discuss below, the neutrinoantineutrino background can be the dominant effect. One goal of this paper is the characterization of neutrino and antineutrino behavior in this nonlinear situation.

The nonlinearity of neutrino oscillations in a dense self-interacting neutrino gas has been addressed by several authors $[8,13,14]$. The correct form of the selfinteraction $[15,16]$ and a scheme for treating the analytical complications involved [17] have been developed only recently. In an earlier work [18], we presented the equations governing the neutrino-antineutrino behavior in the early Universe, incorporating the nonlinear effects of the neutrino-antineutrino background as well as dominant and subdominant effects due to electrons and positrons. We also outlined some results of our numerical simulations to study the general behavior of neutrinos and an- 
tineutrinos in this system.

The present work extends this analysis and provides a more detailed study of the system. For simplicity, we consider a two-flavor model, taking one neutrino to be $v_{e}$ and denoting the other by $v_{\mu}$ (although the latter could represent $v_{\tau}$ instead). The corresponding vacuum masses $m_{1}$ and $m_{2}$ are taken to satisfy $m_{1}<m_{2}$. These choices simplify the parameter space and avoid various complications such as those arising from $C P$ violation in the neutrino mixing matrix. Thus, the $C P$ asymmetry that appears in our analysis has its origin in the small excess of electrons over positrons in the Universe. Similarly, we disregard any differences in the number and energy distributions between the neutrinos and the antineutrinos. As is discussed below, even within these simplifying assumptions, some interesting effects in flavor physics appear. These have their origin in the nonlinear nature of the problem.

The behavior of a particle of energy $E$ and momentum magnitude $p$ propagating in background fields can be described via an index of refraction $n$, given by $E_{\text {eff }}=n p$, where $E_{\text {eff }}$ is the effective particle energy including the interaction energy with the background fields. For a single relativistic neutrino of mass $m$ and specified helicity in vacuo, $n \approx 1+m^{2} / 2 p^{2}$. With two flavors each of two helicities and antiparticles included, the refractive index becomes an $8 \times 8$ matrix. In the presence of a background of electrons, positrons, neutrinos, and antineutrinos, this matrix is modified by the addition of a term representing an expectation of the interaction Hamiltonian in the background. Since only the left-handed neutrinos and right-handed antineutrinos participate in the weak interactions and since different flavors have different behavior, the early Universe is effectively a birefringent medium for neutrinos and antineutrinos. The selfinteractions imply that the birefringence is nonlinear. In effect, this paper characterizes a range of neutrino and antineutrino behavior arising from the nonlinear birefringence.

In Sec. II, we discuss the analytical form of the equations governing neutrino and antineutrino behavior in the early Universe, both in the usual Dirac form and in a density reformulation that we have found more convenient to implement in the simulations. Some details of our numerical methods and more information on our assumptions is provided in Sec. III. The results of the simulations and a description of the general neutrino behavior observed and properties exhibited is given in Sec. IV, along with an analytical approximation scheme for reproducing the gross features of the neutrino behavior. Section IV also includes a separate discussion of the $C P$ asymmetry, which in principle can affect nucleosynthesis or other observable physics. We conclude in Sec. V. For the convenience of the reader, any new phrases or terms we introduce to denote specific neutrino behaviors or properties are written in italics-where they appear for the first time.

\section{EQUATIONS}

In this section, we present the analytical form of the equations we used to describe the behavior of neutrinos in the early Universe. Section II A outlines a derivation of the equations describing neutrino oscillations in background lepton fields. Section II B discusses a reformulation of the results that we have found more useful for numerical work.

\section{A. Oscillation equations}

With the standard assumption of spatial homogeneity and isotropy on the scale of the Hubble volume, the early Universe can be described by the Robertson-Walker metric. At times of interest for our analysis, of order one second or greater, any contribution from the intrinsic curvature is negligible. The effective metric then takes the form

$$
d s^{2}=d t^{2}-a^{2}(t) d \mathbf{x}^{2},
$$

where the time dependence of the scale factor $a(t)$ is governed by the Einstein equations. For a radiationdominated Universe, $a(t) \propto \sqrt{t}$.

As the Universe expands, it cools. The ambient temperature $T_{\gamma}$ of the gas, taken as the photon temperature, is related to the scale factor $a$ by $T_{\gamma} \propto\left(g_{*} a^{3}\right)^{-1 / 3}$. The quantity $g_{*}$ measures the number of effectively massless degrees of freedom. During the radiation-dominated phase, the photon temperature as a function of time is given by

$$
T_{\gamma}=\left(\frac{45}{16 \pi^{3} G_{N} g_{*}}\right)^{1 / 4} \frac{1}{\sqrt{t}},
$$

where $G_{N}$ is Newton's gravitational constant. Prior to the neutrino decoupling, $g_{*}=10.75$ and the neutrino temperature $T_{v}=T_{\gamma}$. After decoupling and electronpositron annihilation, $g_{*}=3.36$ and $T_{v} \approx\left(\frac{4}{11}\right)^{1 / 3} T_{\gamma}$. The combination of the change in $g_{*}$ and the photon reheating means that it is approximately correct to use Eq. (2) with $g_{*}=10.75$ for the neutrino temperature throughout the radiation-dominated era, even after the electronpositron annihilation. In what follows, we denote the neutrino temperature by $T$ for convenience.

The detailed behavior of neutrinos in the early Universe is governed by a Dirac equation appropriate for the Robertson-Walker metric. The main effects arise from the expansion of the scale factor. Intrinsic quantities such as particle number or masses are unaffected by this, but extrinsic ones must be scaled with the comoving volume. For example, number densities scale as $a^{-3}$, while momenta $\mathrm{p}$ scale as $a^{-1}$. Since the relation $E^{2}=\mathrm{p}^{2}+m^{2}$ holds, the energy of relativistic particles effectively also scales as $a^{-1}$.

The presence of the non-Minkowski metric also induces several smaller effects that can be neglected for our purposes. For example, the Dirac spin connection has nonvanishing contributions only from terms that contribute proportional to the Hubble constant $H=\dot{a} / a$, which at a temperature of about $T \sim 1 \mathrm{MeV}$ has a magnitude of order $10^{-22} \mathrm{MeV}$. This means the spin connection can be safely disregarded for values of the neutrino mass parameters we consider.

We are interested in the weak interactions of neutrinos 
with other leptons and with themselves. The effective Hamiltonian governing these processes is a sum of leading terms from charged and neutral current processes:

$$
H=H_{c}+H_{n}+\cdots,
$$

where the ellipsis represents higher-order terms in the Fermi constant $G_{F}$ arising from the expansions of the $W$ and $Z$ propagators. In terms of the fields $e, \mu, v_{e}$, and $v_{\mu}$, respectively, denoting the electron, the muon, the electron neutrino, and the muon neutrino, the explicit form of $H_{c}$ is

$H_{c}=2 \sqrt{2} G_{F}\left(\bar{e}_{L} \gamma^{\lambda} e_{L} \bar{v}_{e L} \gamma_{\lambda} v_{e L}+\bar{\mu}_{L} \gamma^{\lambda} \mu_{L} \bar{v}_{\mu L} \gamma_{\lambda} v_{\mu L}\right)$,

where a superscript $L$ on a fermion denotes the application of the left-handed projector $\left(1-\gamma_{5}\right) / 2$. The explicit form of the relevant terms in $H_{n}$ is

$$
\begin{gathered}
H_{n}=\frac{1}{2} \sqrt{2} G_{F}\left(\bar{v}_{e L} \gamma^{\lambda} v_{e L} \bar{v}_{e L} \gamma_{\lambda} v_{e L}+\bar{v}_{\mu L} \gamma^{\lambda} v_{\mu L} \bar{v}_{\mu L} \gamma_{\lambda} v_{\mu L}\right. \\
\left.+2 \bar{v}_{e L} \gamma^{\lambda} v_{e L} \bar{v}_{\mu L} \gamma_{\lambda} v_{\mu L}\right) .
\end{gathered}
$$

To lowest order in $G_{F}$, quadratic contributions to the neutrino effective Hamiltonian $H_{\text {eff }}$ arising from the presence of background fields can be obtained by replacing pairs of fields in the interaction Hamiltonian $H$ with expectation values. Spatial homogeneity and isotropy implies that only the timelike component of these fourvector expectations are nonzero. The resulting neutrino effective Hamiltonian including the vacuum-mass terms can be written in terms of a $2 \times 2$ matrix $M=M_{m}+M_{b}+M_{\mathrm{fd}}$ :

$H_{\mathrm{eff}}=\frac{G_{F}}{\sqrt{2}}\left(\bar{v}_{e L}, \bar{v}_{\mu L}\right)\left(M_{m}+M_{b}+M_{\mathrm{fd}}\right)\left(\begin{array}{c}v_{e L} \\ v_{\mu L}\end{array}\right)$.

The vacuum-mass contribution $M_{m}$ is given by

$$
M_{m}=\left(\begin{array}{cc}
-\Delta \cos 2 \theta & \Delta \sin 2 \theta \\
\Delta \sin 2 \theta & \Delta \cos 2 \theta
\end{array}\right),
$$

where $\Delta=m_{2}^{2}-m_{1}^{2}$ is the difference of squares of the vacuum masses, while $\theta$ is the vacuum mixing angle between the flavor basis and the mass-eigenstate basis. The background contribution $M_{b}$ is given as

$$
M_{b}=\gamma^{0}\left(\begin{array}{ll}
M_{11} & M_{12} \\
M_{21} & M_{22}
\end{array}\right)
$$

where

$$
M_{11}=-M_{22}=2\left\langle\bar{e}_{L} \gamma_{0} e_{L}\right\rangle+\left\langle\bar{v}_{e L} \gamma_{0} v_{e L}\right\rangle-\left\langle\bar{v}_{\mu L} \gamma_{0} \nu_{\mu L}\right\rangle
$$

and

$$
M_{12}=2\left\langle\bar{v}_{\mu L} \gamma_{0} v_{e L}\right\rangle, \quad M_{21}=2\left\langle\bar{v}_{e L} \gamma_{0} v_{\mu L}\right\rangle
$$

Note that spatial homogeneity implies that $\left\langle\bar{v}_{L} \gamma_{0} v_{L}\right\rangle=n_{v_{L}}-n_{\bar{v}_{L}}$, where $n_{v}$ represents a timedependent but spatially constant neutrino number density. Similarly, the electron expectation is

$$
\left\langle\bar{e}_{L} \gamma_{0} e_{L}\right\rangle=n_{e_{L}^{-}}-n_{e_{L}^{+}} \approx \frac{1}{2}\left(n_{e^{-}}-n_{e^{+}}\right) .
$$

Finally, the contribution $M_{\mathrm{fd}}$ represents flavor-diagonal terms that are irrelevant for oscillations and are disregarded henceforth. In addition to the latter, we have also omitted contributions from the expectation $\left\langle\bar{\mu}_{L} \gamma_{0} \mu_{L}\right\rangle$ because this represents the muon-antimuon numberdensity difference, which is negligible since the muons annihilate at temperatures at least an order of magnitude greater than those relevant for our study.

Equation (6) suffices for the description, exact to order $G_{F}$, of neutrino propagation in the early Universe. Contributions at higher order in $G_{F}$ appear from two sources. One is higher-order neutrino-background interactions arising directly from the four-point interactions in Eqs. (4) and (5). The other is higher-order terms in the expansion of the $W$ and $Z$ propagators in Eq. (3). Most of these terms are several orders of magnitude smaller than the terms in Eq. (6) during the period of interest. However, the electron-positron number-density difference $\sim\left\langle\bar{e}_{L} \gamma_{0} e_{L}\right\rangle$ is a small quantity. Terms at order $G_{F}^{2}$ that contribute as the sum of the electron-positron number densities could therefore be significant. In fact, such terms exist and give an additional contribution $M_{a}$ to the matrix $M$, to be described in Sec. II B below. The numerical analysis discussed in later sections incorporates effects from these terms.

The effective Dirac equation governing the propagation of a left-handed neutrino follows directly from the expression for $H_{\text {eff }}$. The equation is nonlinear due to the presence in $H_{\text {eff }}$ of quantities quadratic in the neutrino fields. A complete analysis requires numerical methods. However, it is more effective to reformulate the above equations in terms of density variables. This is the subject of the next subsection.

\section{B. Reformulation}

Numerical calculations are more readily performed using number-density variables. Let $v_{e}^{j}(t)$ and $v_{\mu}^{j}(t)$ denote the flavor components of the neutrino field with energy $E^{j}$, with similar expressions for the antineutrino field. We rescale the fields by a volume-dependent factor so that the expression $v_{e}^{j^{\dagger}} v_{e}^{j}+v_{\mu}^{j \dagger} v_{\mu}^{j}$ is a constant independent of time. Since only the excess number of electron neutrinos is relevant for oscillations, we choose this constant for convenience to be equal to the excess-neutrino number $N_{v}^{j}$ (discussed in Sec. III below) at energy $E^{j}$ in a canonical comoving volume. A similar condition is taken to hold for the antineutrino fields. In what follows, we use the superscript $j$ for neutrinos, $k$ for antineutrinos, and $i$ for both.

The relevant density variables can be incorporated into vectors $\mathbf{v}^{j}$ and $\mathbf{w}^{k}$ for neutrinos and antineutrinos, respectively $^{1}$ :

\footnotetext{
${ }^{1}$ These vectors play a role analogous to the density matrices $\mathcal{V}^{j}$ and $\mathcal{W}^{k}$ of Ref. [18].
} 


$$
\begin{aligned}
& \mathbf{v}^{j} \equiv\left(v_{e}^{j^{\dagger}} v_{e}^{j}-v_{\mu}^{j \dagger} v_{\mu}^{j}, 2 \operatorname{Re}\left(v_{e}^{j^{\dagger}} v_{\mu}^{j}\right), 2 \operatorname{Im}\left(v_{e}^{j \dagger} v_{\mu}^{j}\right)\right) \\
& \mathbf{w}^{k} \equiv\left(\bar{v}_{e}^{k \dagger} \bar{v}_{e}^{k}-\bar{v}_{\mu}^{k \dagger} \bar{v}_{\mu}^{k}, 2 \operatorname{Re}\left(\bar{v}_{e}^{k \dagger} \bar{v}_{\mu}^{k}\right), 2 \operatorname{Im}\left(\bar{v}_{e}^{k \dagger} \bar{v}_{\mu}^{k}\right)\right)
\end{aligned}
$$

Then, the neutrino-oscillation equations can be rewritten in terms of $\mathbf{v}^{j}$ in a form resembling the motion of a particle in a magnetic field. The same is true for the antineutrino-oscillation equations in terms of $\mathbf{w}^{k}$.

The new equations are $^{2}[18]$

$$
\frac{d \mathbf{v}^{j}}{d t}=\mathbf{v}^{j} \times \mathbf{B}_{v}^{j}, \quad \frac{d \mathbf{w}^{k}}{d t}=\mathbf{w}^{k} \times \mathbf{B}_{w}^{k} .
$$

The "magnetic" fields $\mathbf{B}_{v}^{j}$ and $\mathbf{B}_{w}^{k}$ follow from the equations in the previous subsection. They are given by

$$
\begin{aligned}
& \mathbf{B}_{v}^{j}=\frac{\Delta}{2 E^{j}}-\mathbf{V}_{v v}-\left(V_{C P^{+}}^{j}+V_{C P^{-}}\right) \widehat{\mathbf{e}}_{1}, \\
& \mathbf{B}_{w}^{k}=\frac{\Delta}{2 E^{k}}+\mathbf{V}_{v v}^{*}-\left(V_{C P^{+}}^{k}-V_{C P^{-}}\right) \widehat{\mathbf{e}}_{1} .
\end{aligned}
$$

In these equations,

$$
\Delta=\Delta(\cos 2 \theta,-\sin 2 \theta, 0)
$$

and $\widehat{\mathbf{e}}_{1}=(1,0,0)$. The quantities $V$ are effective potentials that arise from the presence of the background fields.

Defining the action of $C P$ as interchanging neutrino and antineutrino fields without sign change, it follows from Sec. II A that the background electrons and positrons create $C P$-symmetric and $C P$-asymmetric potentials. These are given by [20]

$$
V_{C P^{+}}^{i}=-2 \sqrt{2} G_{F} E^{i}\left(\rho_{e^{-}}+p_{e^{-}}+\rho_{e^{+}}+p_{e^{+}}\right) / M_{W}^{2},
$$

where $\rho$ and $p$ represent energy density and pressure, respectively, and

$$
V_{C P^{-}}=\sqrt{2} G_{F}\left(n_{e^{-}}-n_{e^{+}}\right) .
$$

The $C P$-symmetric potential (14) arises from the additional order- $G_{F}^{2}$ contribution $M_{a}$ to the matrix $M$ of Eq. (6), as mentioned in the previous subsection. Note that higher-order effects in $G_{F}$ arising from $Z$ exchange are omitted from Eq. (14), since they are approximately 2 orders of magnitude smaller than $V_{C P}^{i}$ and hence are negligible for the time period during which our simulations are performed.

Similarly, the potential for the neutrino/antineutrino self-interaction is given by

$$
\mathbf{v}_{v v}=\frac{\sqrt{2} G_{F}}{a^{3}}\left(\langle\mathbf{v}\rangle-\left\langle\mathbf{w}^{*}\right\rangle\right),
$$

where $\langle\mathbf{v}\rangle=\Sigma_{j} \mathbf{v}^{j}$ and $\langle\mathbf{w}\rangle=\Sigma_{k} \mathbf{w}^{k}$ represent the net background values for the neutrino and antineutrino vectors and $a^{3}(t)$ has been selected as the canonical comoving volume in our normalization conventions. The conservation of particle number in a comoving volume at fixed energy is expressed in the reformulated equations as

\footnotetext{
${ }^{2}$ Related equations have recently been given in Ref. [19].
}

$$
\mathbf{v}^{j} \cdot \frac{d \mathbf{v}^{j}}{d t}=0, \quad \mathbf{w}^{k} \cdot \frac{d \mathbf{w}^{k}}{d t}=0 .
$$

Note also that Eqs. (15) and (16) are independent of neutrino/antineutrino energy.

In the above equations, we use an asterisk on a vector to indicate a change of sign of the third component. It follows that the vector $w^{* k}$ obeys the equation

$$
\frac{d \mathbf{w}^{* k}}{d t}=\mathbf{w}^{* k} \times \mathbf{B}_{w^{*}}^{k},
$$

where $\mathbf{B}_{w^{*}}^{k}=-\left(\mathbf{B}_{w}^{k}\right)^{*}$.

The various vector contributions to Eqs. (13) are illustrated in Fig. 1. The four component-vector contributions to $\mathbf{B}_{v}^{j}$, and the vector $\mathbf{B}_{v}^{j}$ itself, are shown (not to scale). The reader should note our choice of orientation of the 1-2-3 axes. A similar figure holds for the vector $\mathbf{B}_{w}^{k}$, but the vector $V_{C P}-\widehat{\mathbf{e}}_{1}$ now points along the positive 1 axis and the nonlinear term $\mathbf{V}_{v v}^{*}$ is reflected in the 1 and 2 axes.

An intuitive understanding of the relative importance of the various terms in Eq. (13) at different times in the early Universe can be found as follows. Associated with each term in the fields $B$ is an effective oscillation time defined as $2 \pi$ over the modulus of that term. We denote these oscillation times as $\tau_{\mathrm{vac}}^{i}$ for the potential involving $\Delta, \tau_{C P^{+}}^{i}$ for the $C P$-symmetric potential, $\tau_{C P^{-}}$for the $C P$-asymmetric potential, and $\tau_{v v}$ for the self-interaction potential. The latter is determined by nonlinear dynamics, but it is always larger than the minimum length

$$
\tau_{v v}^{\min }=2 \pi a^{3} /\left[\sqrt{2} G_{F}(\langle|\mathbf{v}|\rangle+\langle|\mathbf{w}|\rangle)\right],
$$

where $\langle|\mathbf{v}|\rangle=\Sigma_{j}\left|\mathbf{v}^{j}\right|$ and $\langle|\mathbf{w}|\rangle=\Sigma_{k}\left|\mathbf{w}^{k}\right|$. Note that $\langle|\mathbf{v}|\rangle / a^{3}$ and $\langle|\mathbf{w}|\rangle / a^{3}$ represent the excess neutrino and antineutrino number densities, respectively. Comparison of the values of these time scales provides a guide to the dominant effects at any time. Note, in particular, that $\tau_{\mathrm{vac}}^{i}$ and $\tau_{C P}^{i}+$ are energy-dependent quantities.

Figure 2 shows all four time scales as a function of time and temperature in the expanding Universe. We use $\left\langle\tau_{\text {vac }}\right\rangle$ and $\left\langle\tau_{C P^{+}}\right\rangle$to denote the values of $\tau_{\text {vac }}^{i}$ and $\tau_{C P^{+}}^{i}$ averaged over the energy distributions of excess neutrinos

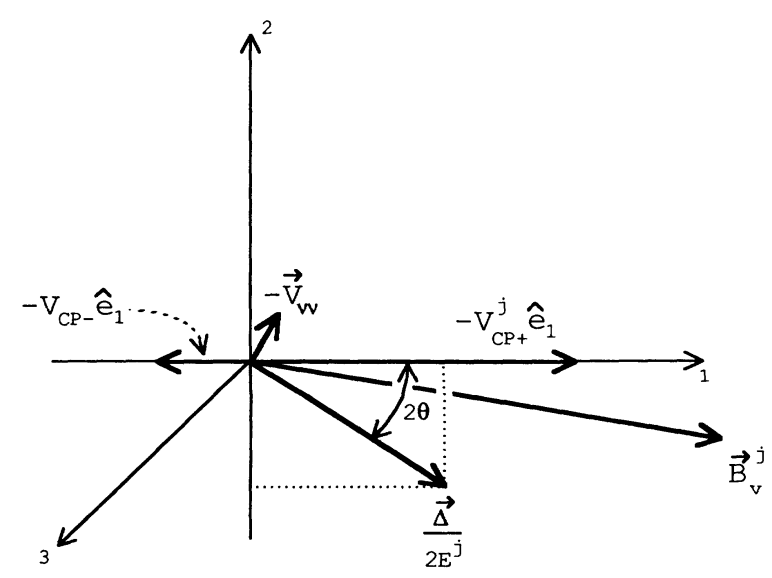

FIG. 1. Components of the vector $\mathbf{B}_{v}^{j}$. 
and antineutrinos. A smaller time scale corresponds to a larger and hence more important potential. The inverse Hubble constant $1 / H$ is also shown. As a time scale moves above the $1 / H$ line, the corresponding term in Eqs. (13) is expected to become unimportant. Note that the $C P$-asymmetric potential $V_{C P^{-}}$is relevant only for temperatures above a few tenths of an $\mathrm{MeV}$, and is smaller than $V_{C P}^{i}$ for all but the lowest energies $E^{i}$.

The figure also displays separately the time scales for electron- and muon-neutrino nonforward scattering, $\left\langle\tau_{v_{e}}^{n f}\right\rangle$ and $\left\langle\tau_{v_{\mu}}^{n f}\right\rangle$, determined from Ref. [20]. The electron neutrinos interact with electrons somewhat more than the muon neutrinos because of the extra contribution from charged currents. As a consequence, electron neutrinos decouple slightly later than muon neutrinos, as indicated in the figure. This means that electron neutrinos participate more in the reheating due to electronpositron annihilation. The higher temperature results in an excess of $\nu_{e}$ over $v_{\mu}$. The associated scales rise above

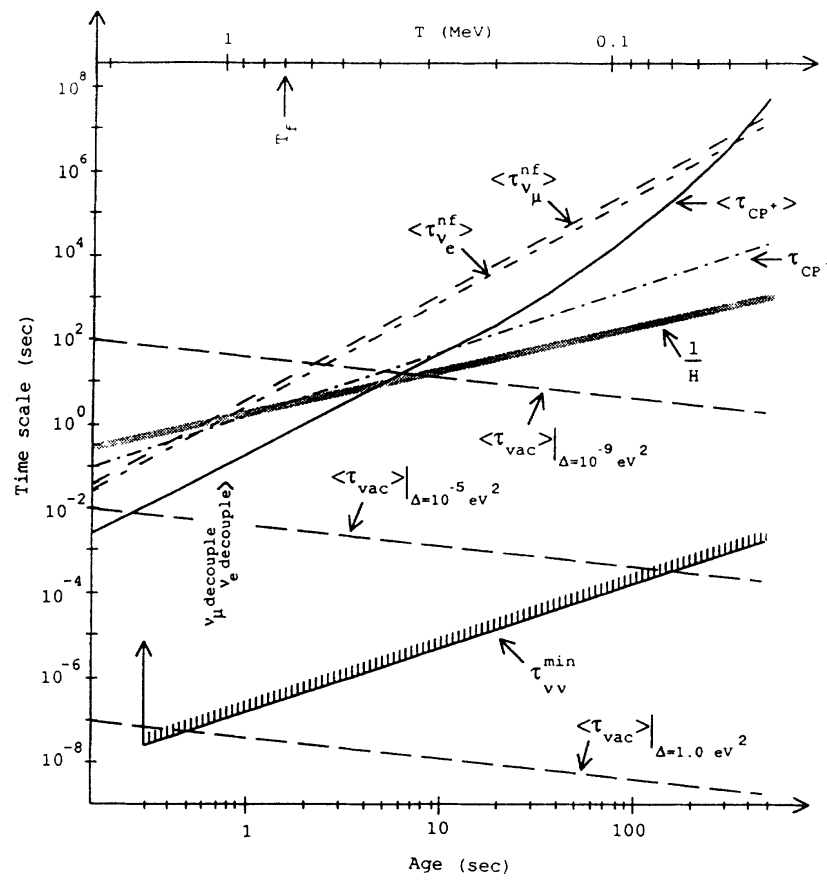

FIG. 2. Relevant time scales. Key time scales are displayed as a function of the age $t$ of the Universe. The time scale $\left\langle\tau_{\mathrm{vac}}\right\rangle$ is displayed for the cases $\Delta=10^{-9}, 10^{-5}$, and $1 \mathrm{eV}^{2}$. For each factor of 10 increase (or decrease) in $\Delta$, the $\left\langle\tau_{\text {vac }}\right\rangle$ line moves down (or up) by one power of ten. The $1 / H$ line is drawn with width as a reminder that it acts as an order-of-magnitude estimate of the time at which a scale becomes insignificant. After the temperature of the Universe has dropped below $T_{f}$, neutrino flavor effects can no longer influence nucleosynthesis. The average nonforward scattering time scales are plotted separately for $v_{e}$ and $v_{\mu}$ for an average energy of $\bar{E}=3.151 T$. The averages $\left\langle\tau_{C P^{+}}\right\rangle$and $\left\langle\tau_{\text {vac }}\right\rangle$ are graphed for an average energy $\bar{E} \approx 5 T$. The arrow on the $\tau_{v v}^{\min }$ line indicates that this quantity becomes effectively infinite at times $t<0.328 \mathrm{~s}$, as a result of our stepfunction approximation for the initial neutrino profile. The hatches on the $\tau_{v v}^{\min }$ line are a reminder that it provides a lower bound for $\tau_{v v}$. the $1 / H$ line during the neutrino-decoupling period, and so nonforward scattering subsequently has little effect. This should be contrasted with neutrino forward scattering off electrons, which is relevant until the $\left\langle\tau_{C P^{+}}\right\rangle$line crosses the $1 / H$ line at an age of several seconds, and neutrino forward scattering off neutrinos, which is relevant until the $\tau_{v v}$ line crosses the $1 / H$ line. The latter time is determined by the nonlinear dynamics. Since for $\tau_{v v}^{\min }$ the crossing time is about a million years, the presence of nonlinear effects can mean neutrino forward scattering off neutrinos is relevant long after the time it becomes negligible in the purely linear case.

It is useful to examine the instantaneous fixed points of Eqs. (12), i.e., subsets of the solution space for which the right-hand sides of Eqs. (12) vanish at a given time. For these points, $\mathbf{v}^{j}$ and $\mathbf{w}^{k}$ would remain constant in time, were it not for the changes in neutrino/antineutrino energies and backgrounds arising from the expansion of the Universe.

Consider first the simpler case where the nonlinear term is absent and the background potentials are time independent. For a given neutrino energy, the mass matrix $M$ in Eq. (6) can then be diagonalized by a $U(2)$ transformation from the flavor basis to the mass-eigenstate basis. The resulting two mass eigenstates correspond to $\mathbf{v}^{j}$ aligned along and against $\mathbf{B}_{v}^{j}$. These orientations evidently generate fixed points since the cross product vanishes. Analogous statements hold for the antineutrinos. Thus, mass eigenstates correspond to fixed points of Eqs. (12) and, once a neutrino is in a mass eigenstate, it remains so thereafter.

When the nonlinear term is present the determination of fixed points is more difficult. At a fixed point, the vectors $\mathbf{v}^{j}$ and $\mathbf{w}^{k}$ must satisfy

$$
\left|\widehat{\mathbf{v}}^{j} \cdot \widehat{\mathbf{B}}_{v}^{j}\right|=1, \quad\left|\hat{\mathbf{w}}^{k} \cdot \widehat{\mathbf{B}}_{w}^{k}\right|=1
$$

where the caret over a quantity denotes a unit vector. However, diagonalization of the matrix $M$ for particles of any one energy changes the background for particles of all other energies and hence can change the alignments of all the other particle vectors. The nonlinearity means that the existence and uniqueness of fixed points is unclear a priori. If $\Delta, V_{C P^{+}}^{i}$ and $V_{C P^{-}}$are all nonzero, one can argue that any fixed points are isolated by comparing the numbers of equations and constraints. However, if $\Delta=V_{C P^{+}}^{i}=V_{C P^{-}}=0$, the system has symmetries leading to continuous families of fixed points. Since, in practice, $\mathbf{V}_{v v}$ can be much larger than the other potentials, there must be continuous subsets of the solution space that lie close to these families of fixed points. This leads to the notion of approximate nonlinear mass-eigenstate (ANME) configurations. In what follows, we call an ANME configuration any configuration of vectors for which Eqs. (19) are approximately valid. It follows that for ANME configurations the time variations of $\mathbf{v}^{j}$ and $\mathbf{w}^{k}$ are relatively slow. Note that, like the linear case, the fixed points are not unique due to the possibility of flipping the signs of $\hat{\mathbf{v}}^{j}$ and $\hat{\mathbf{w}}^{k}$. 


\section{NUMERICAL ANALYSIS}

In this section, we discuss some technical details of the numerical analysis and present information about the simulations performed.

We begin with a discussion of the numerical treatment of the energy distributions of the excess neutrinos and antineutrinos. The excess number-density distribution $f_{v_{e}}(E, T)$ as a function of energy $E$ and temperature $T$, incorporating the spectral distortion due to the neutrino reheating by electron-position annihilation, is given by [3]

$f_{v_{e}}(E, T) \approx\left(6 \times 10^{-4}\right) \frac{E}{T}\left[\frac{11 E}{4 T}-3\right) \frac{E^{2}}{2 \pi^{2}}\left(e^{E / T}+1\right)^{-1}$.

This distribution is shown in Fig. 3. Note that a negative value for $f_{v_{e}}(E, T)$ implies an excess of $v_{\mu}$.

For programming purposes, the continuous energy variable is approximated by a finite number of bins. We have experimented with both constant and variable energy-bin spacing. A sample choice with 50 energy bins and the corresponding values for the distribution at the temperature $1.5 \mathrm{MeV}$ is shown in Table I. The fourth column is obtained using

$$
n_{v}^{j}=f_{v_{e}}\left(E^{j}, T\right) \Delta E^{j},
$$

where $E^{j}$ is the average density in the bin and $\Delta E^{j}$ is the width of the bin.

For definiteness, we choose the factor $a(t)$ to be 1 $\mathrm{MeV}^{-1}$ at the temperature $1.5 \mathrm{MeV}$. Extrinsic quantities are scaled by the appropriate power of $a(t)$. The norms of $\mathbf{v}^{j}$ and $\mathbf{w}^{k}$ are initially equal to the number of excess neutrinos $N_{v}^{i}$ with energy $E^{i}$ in the comoving volume $a^{3}(t)$, i.e.,

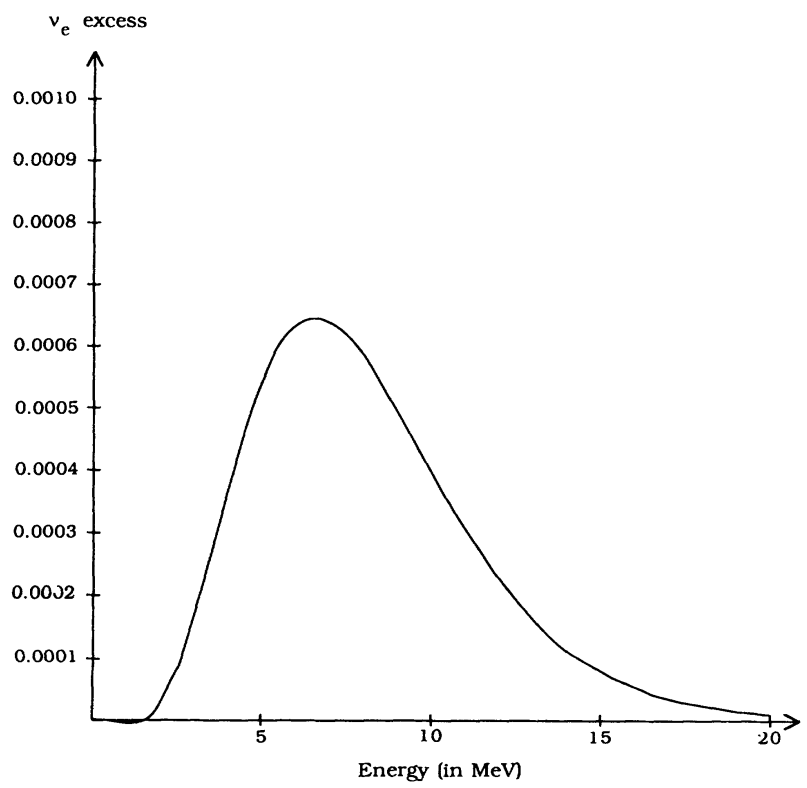

FIG. 3. Neutrino-excess number-density distribution at $T=1.5 \mathrm{MeV}$. At later times, the distribution is the same shape but is scaled.

$$
N_{v}^{i}=\frac{\left|n_{v}^{i}(T=1.5 \mathrm{MeV})\right|}{(1 \mathrm{MeV})^{3}}
$$

Since the norms do not change with time, $\left|\mathbf{v}^{i}\right|$ $=\left|\mathbf{w}^{i}\right|=N_{v}^{i}$ for all times.

The physical $\nu_{e}$ and $\bar{v}_{e}$ excesses are produced over a

TABLE I. Initialization profile. This table displays the bin number, the average energy in the bin, and the excess-neutrino number density as determined at the temperature $T=1.5 \mathrm{MeV}$.

\begin{tabular}{|c|c|c|}
\hline Bin $a$ & $\Delta E^{j}(\mathrm{MeV})$ & $n_{v}^{i}\left(\mathrm{MeV}^{3}\right)$ \\
\hline 1 & 0.0992 & $-1.4304 \times 10^{-9}$ \\
\hline 2 & 0.1555 & $-5.4916 \times 10^{-9}$ \\
\hline 3 & 0.2078 & $-1.0797 \times 10^{-8}$ \\
\hline 4 & 0.2572 & $-1.9606 \times 10^{-8}$ \\
\hline 5 & 0.3068 & $-3.1671 \times 10^{-8}$ \\
\hline 6 & 0.3565 & $-4.7113 \times 10^{-8}$ \\
\hline 7 & 0.4118 & $-8.1638 \times 10^{-8}$ \\
\hline 8 & 0.5914 & $-7.8583 \times 10^{-7}$ \\
\hline 9 & 0.8633 & $-1.9299 \times 10^{-6}$ \\
\hline 10 & 1.6483 & $-6.4468 \times 10^{-7}$ \\
\hline 11 & 2.6393 & $8.4030 \times 10^{-5}$ \\
\hline 12 & 3.2695 & $1.0596 \times 10^{-4}$ \\
\hline 13 & 3.7636 & $1.5700 \times 10^{-4}$ \\
\hline 14 & 4.2595 & $2.0676 \times 10^{-4}$ \\
\hline 15 & 4.7565 & $2.5026 \times 10^{-4}$ \\
\hline 16 & 5.1262 & $1.3855 \times 10^{-4}$ \\
\hline 17 & 5.3759 & $1.4570 \times 10^{-4}$ \\
\hline 18 & 5.6257 & $1.5144 \times 10^{-4}$ \\
\hline 19 & 5.8755 & $1.5576 \times 10^{-4}$ \\
\hline 20 & 6.1002 & $1.2679 \times 10^{-4}$ \\
\hline 21 & 6.3001 & $1.2798 \times 10^{-4}$ \\
\hline 22 & 6.4500 & $6.4237 \times 10^{-5}$ \\
\hline 23 & 6.5500 & $6.4295 \times 10^{-5}$ \\
\hline 24 & 6.7000 & $1.2847 \times 10^{-4}$ \\
\hline 25 & 6.8999 & $1.2784 \times 10^{-4}$ \\
\hline 26 & 7.1247 & $1.5812 \times 10^{-4}$ \\
\hline 27 & 7.3746 & $1.5540 \times 10^{-4}$ \\
\hline 28 & 7.6245 & $1.5189 \times 10^{-4}$ \\
\hline 29 & 7.8744 & $1.4769 \times 10^{-4}$ \\
\hline 30 & 8.1243 & $1.4293 \times 10^{-4}$ \\
\hline 31 & 8.3742 & $1.3771 \times 10^{-4}$ \\
\hline 32 & 8.7462 & $2.5839 \times 10^{-4}$ \\
\hline 33 & 9.2456 & $2.3435 \times 10^{-4}$ \\
\hline 34 & 9.7451 & $2.0972 \times 10^{-4}$ \\
\hline 35 & 10.2446 & $1.8546 \times 10^{-4}$ \\
\hline 36 & 10.7442 & $1.6228 \times 10^{-4}$ \\
\hline 37 & 11.2438 & $1.4063 \times 10^{-4}$ \\
\hline 38 & 11.7435 & $1.2081 \times 10^{-4}$ \\
\hline 39 & 12.2432 & $1.0297 \times 10^{-4}$ \\
\hline 40 & 12.7429 & $8.7125 \times 10^{-5}$ \\
\hline 41 & 13.4701 & $1.3440 \times 10^{-4}$ \\
\hline 42 & 14.4682 & $9.2789 \times 10^{-5}$ \\
\hline 43 & 15.4666 & $6.2763 \times 10^{-5}$ \\
\hline 44 & 16.8591 & $6.8988 \times 10^{-5}$ \\
\hline 45 & 19.3944 & $4.0387 \times 10^{-5}$ \\
\hline 46 & 22.8374 & $4.3994 \times 10^{-6}$ \\
\hline 47 & 25.3284 & $2.2322 \times 10^{-6}$ \\
\hline 48 & 29.2997 & $2.8185 \times 10^{-7}$ \\
\hline 49 & 33.2780 & $3.2936 \times 10^{-8}$ \\
\hline 50 & 37.2611 & $3.6257 \times 10^{-9}$ \\
\hline
\end{tabular}
The total excess is $4.84 \times 10^{-3}$ neutrinos per $\mathrm{MeV}^{-3}$. 
period of approximately $\frac{1}{2} s[2,3]$. In the early stages of the production few neutrinos are present, so the nonlinear potential in Eqs. (13) is negligible. These early produced neutrinos start in flavor eigenstates and then begin to oscillate about mean values set by linear mass eigenstates. The oscillation time scale is about 2 orders of magnitude greater than the production time scale. Therefore, neutrinos introduced into the system at different times are out of phase. As more particles are produced, the background grows. Provided that nonlinear effects from this background do not counteract the decoherence, the final neutrino profile is likely to be an ANME configuration.

For the present simulations, we have approximated the continuous production process by an instantaneous one occurring at a temperature of $1.5 \mathrm{MeV}(t \approx 0.328 \mathrm{~s})$ in ANME configurations. A useful method of constructing these numerically is to begin with analytical mass eigenstates found by setting the terms $\mathbf{V}_{v v}$ and $V_{C P^{-}}$to zero (motivation for this is given in Sec. IV D). An iterative procedure with all four potential terms present then can generate ANME configurations satisfying Eqs. (19) to machine accuracy.

To check sensitivity to initial conditions, we have also performed simulations with neutrinos initially in flavor eigenstates. As is discussed in Sec. IV A below, the simulations suggest that neutrino behavior is insensitive to initial conditions for $1 \mathrm{eV}^{2} \lesssim \Delta$ and $\Delta \lesssim 10^{-7} \mathrm{eV}^{2}$. For other values of $\Delta$, initialization in an ANME configuration provides a best guess. However, the reader should be aware that numerical implementation of the physical production mechanism could conceivably generate a different effective initial configuration, such as an ANME configuration rotated slightly toward the flavor axis.

More explicitly, the values of the potentials $V_{C P}^{i}$ and $V_{C P^{-}}$were given according to Eqs. (14) and (15) and are

$$
\begin{gathered}
V_{C P^{+}}^{i}=-\frac{2 \sqrt{2} G_{F} E^{i}}{M_{W}^{2}} \frac{4}{6 \pi^{2}} \int_{m_{e}}^{\infty} \\
d E\left(E^{2}-m_{e}^{2}\right)^{1 / 2}\left(4 E^{2}-m_{e}^{2}\right) \\
\times\left(e^{E / T} \gamma+1\right)^{-1}
\end{gathered}
$$

and

$$
V_{C P^{-}}=0.9 \eta \frac{2 \xi(3)}{\pi^{2}} T_{\gamma}^{3},
$$

where $m_{e}$ is the electron mass, $M_{W}$ is the $W$-boson mass, and the $\zeta$ function is $\zeta(3)=1.20206$. . The integral in Eq. (23) arises from the combination of energy and pressure densities in Eq. (14). In practice, we replaced the full integral with a phenomenological approximation that reproduced values of the integral to the necessary precision. In Eq. (24), the numerical factor of 0.9 represents the percentage of baryons that are protons and hence measures the net excess number of electrons in the Universe. Also, $\eta$ is the baryon-to-photon ratio, which we took to be $4 \times 10^{-10}$. The remaining factors merely count the number of photons at the temperature $T_{\gamma}$. Note that our discussions about neutrino behavior in this paper should be relatively insensitive to numerical uncertainties in parameters such as $\eta$ or the coefficient
$6 \times 10^{-4}$ in Eq. (20) because the scale for neutrino selfinteractions is widely separated from the other scales (cf. Fig. 2).

With $N$ energy bins, Eqs. (12) become a set of $2 N$ coupled differential equations. Knowing the initialization profiles, we can then integrate these equations numerically for each energy-bin vector. We used a fourth-order Runge-Kutta method with a variable time step, selected at each step to be several orders of magnitude smaller than the smallest relevant oscillation time at that step.

The simulations were performed on an HP Apollo Series 700 workstation. We studied a region of the $\sin ^{2} 2 \theta-\Delta$ plane given by $10^{-20} \mathrm{eV}^{2}<\Delta<10^{2} \mathrm{eV}^{2}$ and $-\pi / 2<2 \theta<+\pi / 2$. In particular, we focused on selected values of $\sin ^{2} 2 \theta$, including a "medium" value of 0.25 and a "large" value of 0.81 . Small values lead to little flavor evolution and are therefore of lesser interest. For the reader who may wish to examine unexplored areas of the plane, the list of values we have studied are listed in Table II.

Previous treatments of neutrino oscillations in the early Universe neglect the terms $V_{C P^{+}}^{i}$ and $\mathbf{V}_{v v}$. Under this assumption, a Mikheyev-Smirnov-Wolfenstein- (MSW-) type conversion [21] for neutrinos (but not antineutrinos) occurs for $\Delta$ around $10^{-7} \mathrm{eV}^{2}$. From Fig. 2, one sees that $\left\langle\tau_{\text {vac }}\right\rangle$ and $\tau_{C P^{-}}$cross at $T \sim 1 \mathrm{MeV}$, so the vacuum term containing $\Delta$ partially cancels the potential $V_{C P^{-}}$in Eqs. (13). At earlier times $V_{C P^{-}}$is larger so $\mathbf{B}_{v}^{j}$ points almost along $-\widehat{\mathbf{e}}_{1}$, while at later times the vacuum term dominates and $\mathbf{B}_{v}^{j}$ points along $\hat{\Delta}$. This means $\mathbf{B}_{v}^{j}$ undergoes a large rotation. Provided the rotation is slow relative to the neutrino-oscillation time scale, the neutrino vectors can follow $\mathbf{B}_{v}^{j}$, resulting in a large flavor conversion [9]. Our test simulations using only $V_{C P^{-}}$and the vacuum term containing $\Delta$ verify that this occurs.

In contrast, with $V_{C P^{j}}^{j}$ present only a small neutrino flavor conversion occurs for $\Delta$ around $10^{-7} \mathrm{eV}^{2}$. The vector $\mathbf{B}_{v}^{j}$ undergoes a much smaller rotation because at earlier times it now points almost along $+\hat{\mathbf{e}}_{1}$ instead of

TABLE II. Runs performed.

\begin{tabular}{lc}
\hline \hline$\Delta\left(\mathrm{eV}^{2}\right)$ & $\sin ^{2} 2 \theta$ \\
\hline $1.0 \times 10^{-20}$ & $0.25,0.81$ \\
$1.0 \times 10^{-15}$ & $0.01,0.25,0.81$ \\
$1.0 \times 10^{-14}$ & 0.81 \\
$1.0 \times 10^{-12}$ & $0.25,0.81$ \\
$1.0 \times 10^{-10}$ & $0.25,0.81$ \\
$5.0 \times 10^{-10}$ & 0.25 \\
$1.0 \times 10^{-9}$ & $0.14,0.18,0.25,0.81$ \\
$2.0 \times 10^{-9}$ & $0.040,0.14,0.18,0.25$ \\
$3.0 \times 10^{-9}$ & $0.14,0.25$ \\
$8.0 \times 10^{-9}$ & 0.14 \\
$1.0 \times 10^{-8}$ & $0.25,0.81$ \\
$1.0 \times 10^{-7}$ & $0.25,0.81$ \\
$1.0 \times 10^{-6}$ & $0.25,0.81$ \\
$1.0 \times 10^{-5}$ & $0.25,0.81$ \\
$1.0 \times 10^{-4}$ & $0.25,0.81$ \\
$1.0 \times 10^{+2}$ & $0.25,0.81$ \\
\hline \hline
\end{tabular}


$-\widehat{\mathbf{e}}_{1}$ since $\left|V_{C P}^{j}\right|>\left|V_{C P^{-}}\right|$. However, for neutrinos with energies less than about 20 times the average energy, $\left|V_{C P^{+}}^{j}\right|<\left|V_{C P^{-}}\right|$and a large flavor conversion can occur at around $T=1 \mathrm{MeV}$ if $\Delta / 2 E^{j}$ is slightly smaller than the difference $\left|V_{C P^{-}}\right|-\left|V_{C P^{+}}^{j}\right|$, i.e., for $\Delta \sim 3 \times 10^{-9} \mathrm{eV}^{2}$. Since this low-energy $M S W$ conversion has the potential to lead to interesting effects, we have carried out more simulations in this parameter region; cf. Table II.

\section{RESULTS AND DISCUSSION}

In this section, we present details of our results and discuss some features we have identified. Section IV A provides an overview of neutrino behavior in different regions of the $\sin ^{2} 2 \theta-\Delta$ plane. We have found that the oscillations obey certain properties, which are described in Sec. IV B. The issues of $C P$ asymmetry and its possible consequences are considered in Sec. IV C. Finally, in Sec. IV D we present an analytical approximation scheme for calculating the neutrino and antineutrino density vectors.

\section{A. Overview of neutrino behavior}

For convenience in what follows, we identify four regions of the $\sin ^{2} 2 \theta-\Delta$ plane. They are

region $1(\mathrm{R} 1)$ : $1 \mathrm{eV}^{2} \lesssim \Delta$;

region $2(\mathrm{R} 2): 10^{-5} \mathrm{eV}^{2} \lesssim \Delta \lesssim 1 \mathrm{eV}^{2}$;

region $3(\mathrm{R} 3): 10^{-9} \mathrm{eV}^{2} \lesssim \Delta \lesssim 10^{-5} \mathrm{eV}^{2}$;

region 4 (R4): $\Delta \lesssim 10^{-9} \mathrm{eV}^{2}$.

In $R 1$, the lepton gas is insufficiently dense to have an appreciable effect. The vacuum terms in Eqs. (12) dominate the potentials due to the background fields and so the system behaves in a manner similar to the vacuum case. Any ANME configurations are close to vacuummass eigenstates. If the initial neutrino/antineutrino configuration is in flavor eigenstates, then within a few oscillation times decoherence appears and the average vectors $\langle\mathbf{v}\rangle$ and $\langle\mathbf{w}\rangle$ effectively align themselves along the direction $\hat{\Delta}$ of vacuum-mass eigenstates. The point is that the distribution of energies implies a distribution of oscillation times, so even if the neutrinos begin in phase, they soon decohere.

To be more specific, consider the intrinsic ratio

$$
\mathbf{r}_{v}=\langle\mathbf{v}\rangle /\langle|\mathbf{v}|\rangle
$$

where

$$
\langle|\mathbf{v}|\rangle=\sum_{j}\left|\mathbf{v}^{j}\right|=\sum_{j} N_{v}^{j}=N_{v}
$$

and $N_{v}$ is the total number of neutrinos in the comoving volume. The presence of the denominator ensures that the components of $\mathbf{r}_{v}$ range between -1 and 1 . It also means that uncertainties arising from our approximations and from imprecisely known parameters tend to cancel. Initially, $\mathbf{r}_{v} \approx(0.9985,0,0)$ rather than $(1,0,0)$ due to the small muon-neutrino excess at small energies. The asymptotic value of $\mathbf{r}_{v}$ is

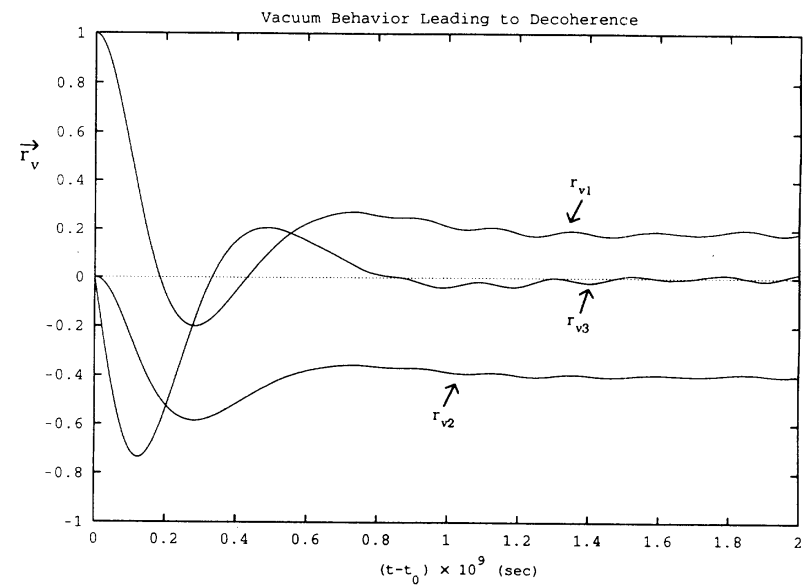

FIG. 4. Vacuum behavior leading to decoherence. The ratios $\mathbf{r}_{v}$ are displayed as a function of time for $\Delta=10^{2} \mathrm{eV}^{2}$ and $\sin ^{2} 2 \theta=0.81$.

$$
\mathbf{r}_{v} \approx \cos 2 \theta(\cos 2 \theta,-\sin 2 \theta, 0) \text {. }
$$

This is readily understood: $\mathbf{r}_{v}$ points asymptotically along $\hat{\boldsymbol{\Delta}}$. The projection factor of $\cos 2 \theta$ is the same as the dot product of the flavor direction $(1,0,0)$ and $\hat{\Delta}$. It appears because the vectors $\mathbf{v}^{j}$ circle around $\widehat{\Delta}$ and eventually spread into a cone due to the decoherence.

More generally, if the vectors $\mathbf{v}^{j}$ initially all point in one direction at an angle $\alpha$ with respect to $\widehat{\Delta}$, then asymptotically

$$
\mathbf{r}_{v} \approx \cos \alpha(\cos 2 \theta,-\sin 2 \theta, 0) .
$$

If, instead, the initial vectors form an ANME configuration, then asymptotically $\mathbf{r}_{v} \approx(\cos 2 \theta,-\sin 2 \theta, 0)$ because $\cos \alpha \approx 1$.

In analogy with Eq. (25), a ratio $\mathbf{r}_{w}$ can be defined for the antineutrino vectors. In what follows, our figures do not display $\mathbf{r}_{w}$ for the nonlinear case because it is indistinguishable from $\mathbf{r}_{v}$ at the scales used. Also, we often do not show $r_{v 3}$ because it is close to zero.

Figure 4 displays $\mathbf{r}_{v}$ for $\Delta=10^{2} \mathrm{eV}^{2}$ and $\sin ^{2} 2 \theta=0.81$. Decoherence is apparent after one "bounce." The asymptotic value of $\mathbf{r}_{v} \approx(0.19,-0.39,0)$ is achieved.

In R2, neutrino behavior is sensitive to initial conditions. If the initial configuration represents flavor eigenstates, then self-maintained coherence [17] arises: a collective mode of the system appears in which some fraction of the particles oscillate in phase. ${ }^{3}$ An example of this behavior is shown in Figs. 5 and 6, which display components of the ratio $\mathbf{r}_{v}$ versus time. Regular periodic behavior occurs, as the neutrino vectors oscillate almost in the 1-2 plane about the direction of the ANME configuration. The neutrino vectors remain bunched together and move more or less as a single unit.

\footnotetext{
${ }^{3}$ The coherence is in flavor space. Quantum decoherence is automatic since the neutrino wave functions have random phases. Note that any overall phase for a neutrino cancels in Eq. (11) and does not affect the oscillations we consider.
} 


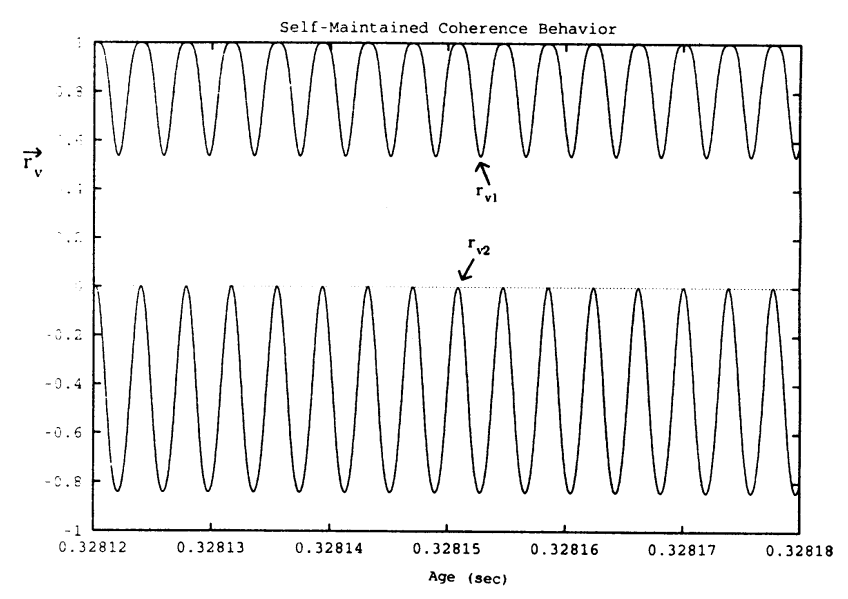

FIG. 5. Self-maintained coherence. The ratios $r_{v 1}$ and $r_{v 2}$ are displayed as a function of time for $\Delta=10^{-4} \mathrm{eV}^{2}$ and $\sin ^{2} 2 \theta=0.25$.

This behavior can be attributed to the presence of the nonlinear potential term $\mathbf{V}_{v v}$ in the first of Eqs. (13). The motion can be understood via its third component $V_{v v 3}$, proportional to $\left\langle v_{3}\right\rangle-\left\langle w_{3}^{*}\right\rangle$. As can be seen from Fig. 6 , this quantity periodically changes sign, thereby creating an alternating "magnetic" field along the third direction that induces the back-and-forth planar behavior. The reason why $V_{v v 3}$ initially acquires a negative value is discussed below (the "rotation" mechanism). Once this has occurred, the vectors $\mathbf{v}^{j}$ and $\mathbf{w}^{k}$ turn toward $\Delta$, eventually overshooting it. At that point, $V_{v v 3}$ begins to increase and subsequently turns positive. This then induces the vectors $\mathbf{v}^{j}$ and $\mathbf{w}^{k}$ to swing back towards $\Delta$, and the process thereby continues indefinitely. Coherence is maintained, i.e., neutrinos of different energy move together, because $V_{v v 3}$ is independent of energy.

For this region of the $\sin ^{2} 2 \theta-\Delta$ plane, any selfmaintained coherence eventually disappears when the $\left\langle\tau_{\text {vac }}\right\rangle$ line crosses the $\tau_{v v}^{\min }$ line, whereupon the nonlinear term becomes insufficiently strong to maintain coherent behavior. Vacuum behavior then arises instead. This effect is illustrated in Fig. 7. The self-maintained

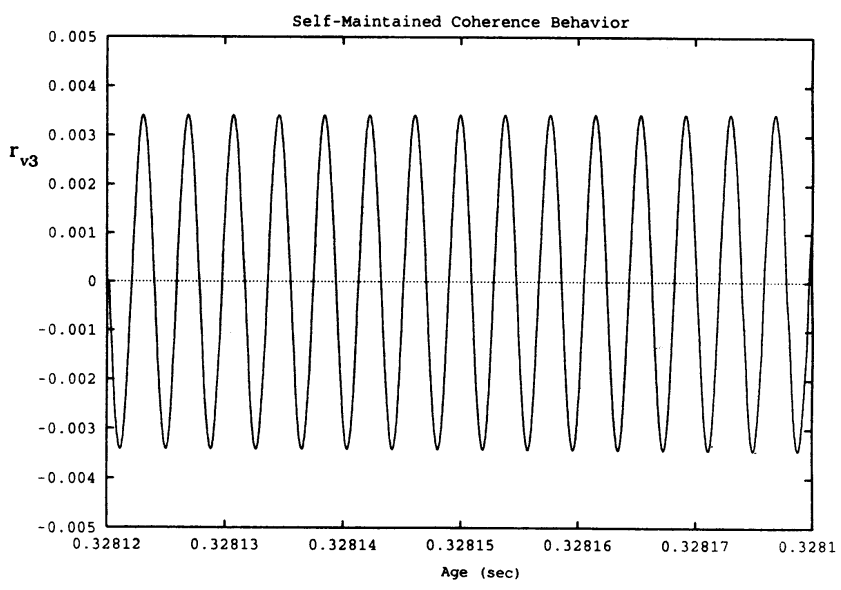

FIG. 6. The ratio $r_{v 3}$ as a function of time for $\Delta=10^{-4} \mathrm{eV}^{2}$ and $\sin ^{2} 2 \theta=0.25$. Note the relatively small scale of the vertical axis.

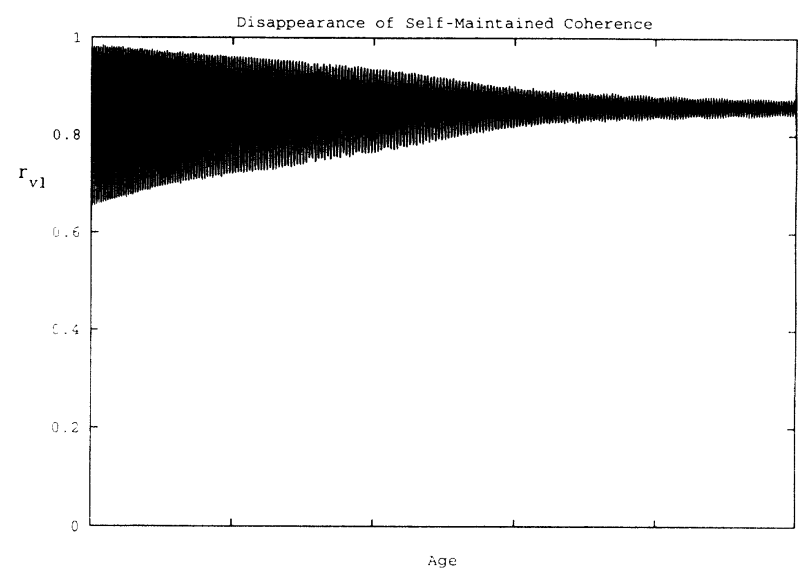

FIG. 7. Disappearance of self-maintained coherence. The small amplitude eventually shrinks to zero.

coherent oscillations gradually shrink in amplitude. Lower-energy particles decohere before higher-energy ones, so the process exhibits some continuity. In this particular example, decoherence sets in only after many oscillation times.

If the physical production mechanism leads instead to an effective ANME configuration at our initialization point that is slightly rotated toward the flavor axis, then self-maintained coherence occurs with a smaller amplitude. The general features of this behavior are similar to those discussed above. In what follows, we turn our attention to the case where there is initially an ANME configuration.

In $\mathrm{R} 2$, simulations were practical only for $\Delta=10^{-4}$ and $10^{-5} \mathrm{eV}^{2}$. For $\Delta>10^{-4} \mathrm{eV}^{2}$ it is desirable to extend the simulation to a time of approximately $6 \mathrm{~s}$, where $V_{C P^{-}}$and $V_{C P^{+}}^{i}$ become negligible (cf. Fig. 2). However, at $\Delta=10^{-4} \mathrm{eV}^{2}$ this involves more than 10000 oscillation times, and each tenfold increase in $\Delta$ increases the number of oscillation times by an order of magnitude. The buildup of numerical error in the integration algorithm therefore becomes an important issue.

For manageable values of $\Delta$, we observed little motion away from ANME configurations. This held true for several thousand oscillation times. The plots of the three components of $\mathbf{r}_{v}$ are merely three horizontal lines, so we do not display them. Unless parametric effects arise, the behavior is rather mundane. We also expect this behavior to occur elsewhere in R2, modulo possible parametric effects.

One parametric effect could be self-maintained coherence. Fluctuations in the background may orient some fraction of the particles away from an ANME configuration, thereby inducing small-amplitude selfmaintained coherence. Deciding this issue and uncovering any other parametric effects requires more computer power.

In R3 and R4, the direction of an ANME configuration rotates with time and the particles maintain alignment with it. They begin near flavor eigenstates and smoothly convert to vacuum-mass eigenstates. We refer to this behavior as evolutionary. It is achieved by rota- 
tion rather than decoherence. The rotation occurs in the region where the $\left\langle\tau_{\text {vac }}\right\rangle$ line crosses the $\left\langle\tau_{C P^{+}}\right\rangle$line in Fig. 2.

Figures 8-11 plot lines of constant $r_{v 1}$ and $r_{v 2}$ for $\sin ^{2} 2 \theta=0.25$ and 0.81 . With a fixed value of $\Delta$ and the corresponding $\left\langle\tau_{\mathrm{vac}}\right\rangle$ line drawn on the figure, the contours provide the values of $r_{v 1}$ and $r_{v 2}$ at different times. Lines of this type are explicitly drawn on the figures for $\Delta=10^{-12}, 10^{-9}, 10^{-6}$, and $10^{-4} \mathrm{eV}^{2}$. The ratio $\mathbf{r}_{v}$ is near $(1,0,0)$ in the upper left of all four figures and becomes $(\cos 2 \theta,-\sin 2 \theta, 0)$ in the lower right, corresponding to late times for which $\left\langle\tau_{\mathrm{vac}}\right\rangle$ drops sufficiently below $\left\langle\tau_{C P}{ }^{+}\right\rangle$. The marked points correspond to canonical choices that are listed in Table III; see Sec. IV B. Note that the contour lines are almost parallel to the $\left\langle\tau_{C P^{+}}\right\rangle$ line of Fig. 2.

The reasons underlying the evolutionary behavior differ in R3 and R4. In R3, the rotation occurs adiabatically, i.e., any change in the direction of the ANME configurations during an oscillation time is small. To gain insight, it is useful to consider first the linear case, in which $\mathbf{V}_{v v}$ is set to zero and for which the mass eigenstates lie in the 1-2 plane. Simulations for this case also display rotation of the particle vectors from flavor eigenstates to vacuum-mass eigenstates as the region near the $\left\langle\tau_{C P^{+}}\right\rangle$line of Fig. 2 is traversed. The neutrinos and antineutrinos undergo adiabatic rotation, except perhaps for low-energy neutrinos in certain restricted regions of parameter space that convert via an MSW-type effect. Since the various potentials have power-law behavior with time, the relevant time scale for the change in the instantaneous matter-induced mixing angle is the Hubble constant. Hence, as long as the oscillation time is smaller than $1 / H$ the motion is adiabatic. As can be seen in Fig. 2 , this is true for $10^{-9} \mathrm{eV}^{2} \lesssim \Delta$.

Unlike the linear case where a single angle determines

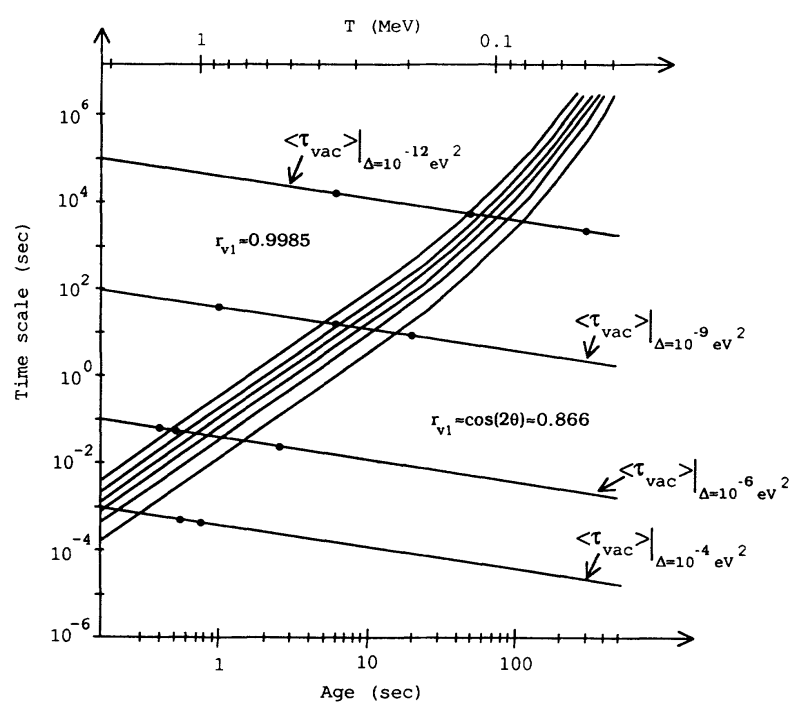

Lines of Constant $r_{v 1}$

FIG. 8. Lines of constant $r_{v 1}$ for $\sin ^{2} 2 \theta=0.25$. From top to bottom, the contours displayed are for constant $r_{v 1}=0.98,0.96$, $0.94,0.92,0.90$, and 0.88 .

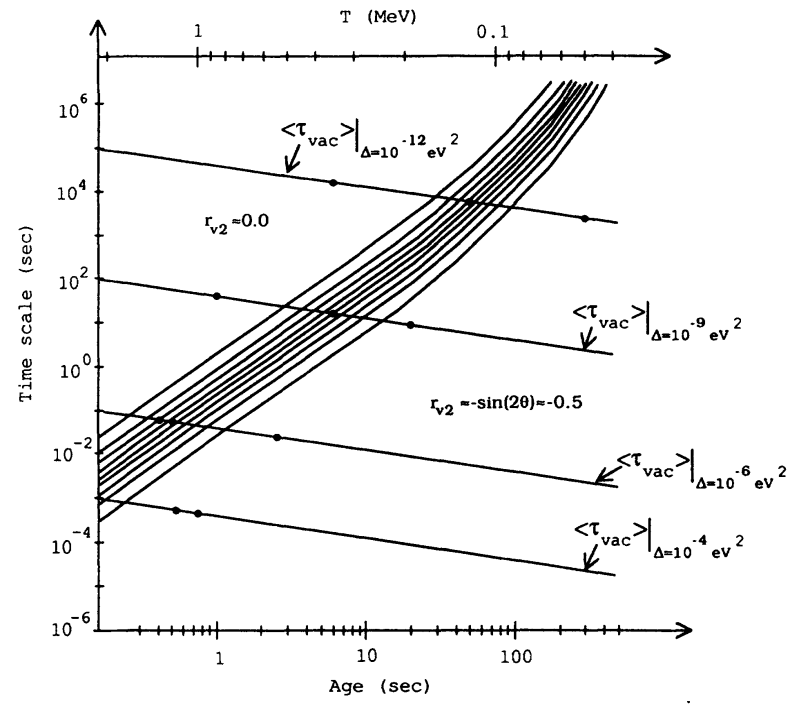

Lines of Constant $r_{v 2}$

FIG. 9. Lines of constant $r_{v 2}$ for $\sin ^{2} 2 \theta=0.25$. From top to bottom, the contours displayed are for constant $r_{v 2}=-0.05$, $-0.10,-0.15,-0.20,-0.25,-0.30,-0.35,-0.40$, and -0.45 .

the mass eigenstate basis, two angles are needed when the nonlinear term $\mathbf{V}_{v v}$ is present. This is easily seen in our vector reformulation: any ANME configurations are determined by a direction in three-space (cf. Fig. 1). Hence, the definition of adiabaticity used in the linear case must be generalized. One might be tempted to choose as a working definition

$$
2 \pi \frac{\left|d \mathbf{B}^{i} / d t\right|}{\left|\mathbf{B}^{i}\right|} \ll 1
$$

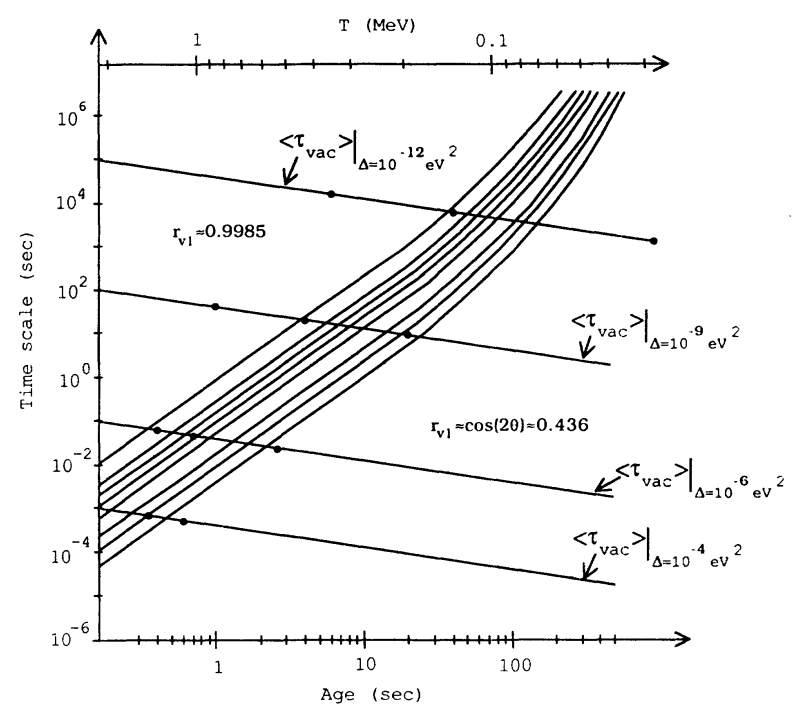

Lines of Constant $r_{v 1}$

FIG. 10. Lines of constant $r_{v 1}$ for $\sin ^{2} 2 \theta=0.81$. From top to bottom, the contours displayed are for constant $r_{v 1}=0.98,0.9$, $0.8,0.7,0.6,0.5,0.47$, and 0.45 . 


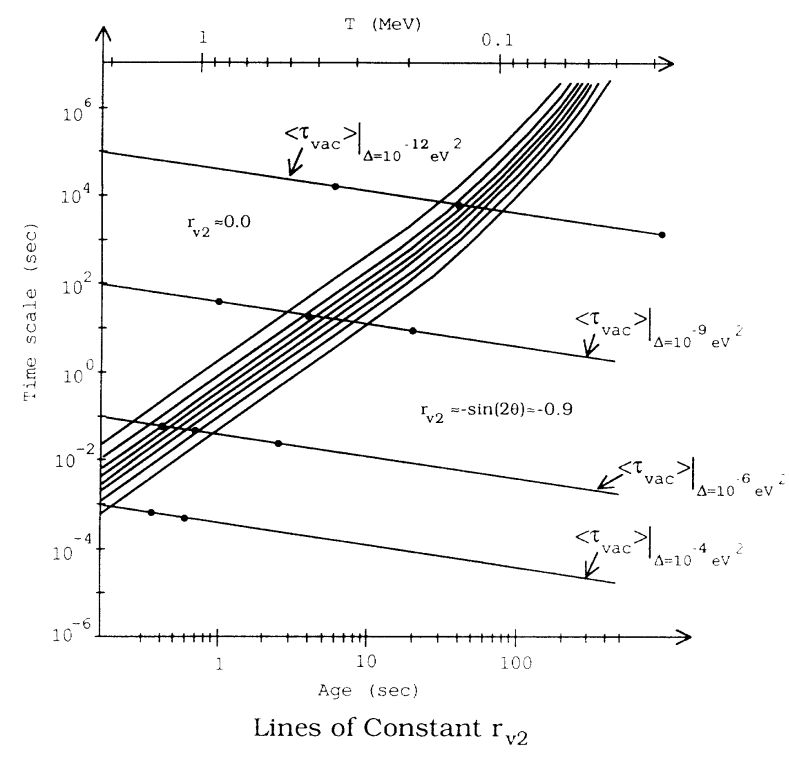

FIG. 11. Lines of constant $r_{v 2}$ for $\sin ^{2} 2 \theta=0.81$. From top to bottom, the contours displayed are for constant $r_{v 2}=-0.1$, $-0.2,-0.3,-0.4,-0.5,-0.6,-0.7$, and -0.8 .

as the adiabaticity condition. When this condition is satisfied, the change of direction of $\mathbf{B}$ is small during an oscillation time $2 \pi /|\mathbf{B}|$, so the particle vectors can follow the motion.

For $\Delta \lesssim 10^{-8} \mathrm{eV}^{2}$ the behavior of the linear and nonlinear cases is similar. Figure 12 compares the first two components of the ratio $\mathbf{r}_{v}$ at $\Delta=10^{-8} \mathrm{eV}^{2}$. For $\Delta \lesssim 10^{-7}$

TABLE III. Canonical values. This table lists canonical points for the discussion in the text. At $\Delta=10^{-4} \mathrm{eV}^{2}$, there is no $4 b$ run because the crossover region is not traversed. The canonical points are marked in Figs. 8-11.

\begin{tabular}{cccr}
\hline \hline $\sin ^{2} 2 \theta$ & Point & $\Delta\left(\mathrm{eV}^{2}\right)$ & Time $(\mathrm{s})$ \\
\hline 0.25 & $12 a$ & $10^{-12}$ & 6.00047 \\
& $12 b$ & $10^{-12}$ & 50.00140 \\
$12 c$ & $10^{-12}$ & 300.00417 \\
$9 a$ & $10^{-9}$ & 1.00000 \\
& $9 b$ & $10^{-9}$ & 6.00000 \\
$9 c$ & $10^{-9}$ & 20.000000 \\
& $6 a$ & $10^{-6}$ & 0.40000 \\
$6 b$ & $10^{-6}$ & 0.51100 \\
& $6 c$ & $10^{-6}$ & 2.55722 \\
& $4 a$ & $10^{-4}$ & 0.54239 \\
& $4 c$ & $10^{-4}$ & 0.76368 \\
0.81 & $12 a$ & $10^{-12}$ & 6.00021 \\
& $12 b$ & $10^{-12}$ & 40.02332 \\
& $12 c$ & $10^{-12}$ & 900.00357 \\
$9 a$ & $10^{-9}$ & 1.00001 \\
& $9 b$ & $10^{-9}$ & 4.00005 \\
& $9 c$ & $10^{-9}$ & 20.00003 \\
$6 a$ & $10^{-6}$ & 0.40000 \\
& $6 b$ & $10^{-6}$ & 0.70000 \\
$6 c$ & $10^{-6}$ & 2.69253 \\
& $4 a$ & $10^{-4}$ & 0.35000 \\
& $4 c$ & $10^{-4}$ & 0.59665 \\
\hline \hline
\end{tabular}

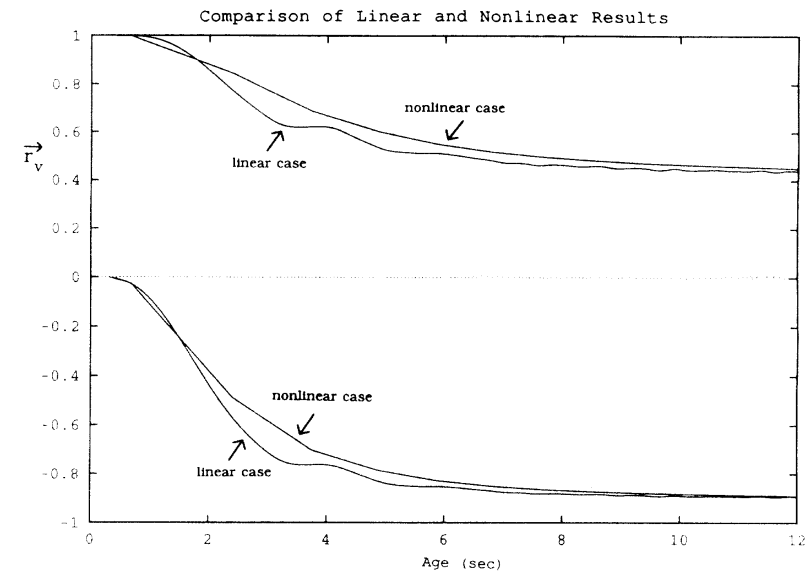

FIG. 12. Comparison of linear and nonlinear results. The ratios $r_{v 1}$ and $r_{v 2}$ are displayed as a function of time for $\Delta=10^{-8}$ $\mathrm{eV}^{2}$ and $\sin ^{2} 2 \theta=0.81$.

$\mathrm{eV}^{2}$ the two cases are indistinguishable at this scale. For this reason, we have not used our canonical value of $\Delta=10^{-6} \mathrm{eV}^{2}$.

During the simulations with the nonlinear term we checked the condition (26) and found that it was not always satisfied. The motion is evidently adiabatic despite this. It turns out that, although the nonlinear term $\langle\mathbf{v}\rangle-\left\langle\mathbf{w}^{*}\right\rangle$ is small, it fluctuates rapidly, thereby inducing violations of the condition (26). However, rapid fluctuations with a tiny amplitude should not ruin adiabaticity. An analogy can be made to the case of an adiabatic MSW effect in the Sun: at distance scales comparable to the electron spacing, an individual neutrino sees a highly fluctuating potential that is small away from the electrons. Nonetheless, adiabaticity is valid. For this reason, we choose to replace Eq. (26) with

$$
2 \pi \frac{\overline{\left|d \mathbf{B}^{i} / d t\right|}}{\left|\mathbf{B}^{i}\right|}<<1 .
$$

The bar indicates a time averaging performed over a fraction of an oscillation time. Equation (27) is our definition of the adiabatic condition. In R3 and R4, the neutrino vectors undergo changes in a relatively narrow region called the crossover region (see, for example, Figs. 8-11). Over the whole of R3, we found that the condition (27) was satisfied in the crossover region.

In R4 our adiabaticity condition (27) is violated in the crossover region, as expected. Nonetheless, particles exhibit evolutionary behavior from flavor to vacuum-mass eigenstates. However, the transition occurs significantly before $\left\langle\tau_{\text {vac }}\right\rangle$ becomes smaller than $1 / H$.

A new type of behavior due to the term $\left\langle v_{3}\right\rangle-\left\langle w_{3}^{*}\right\rangle$ is responsible. We call it the rotation mechanism, and it works as follows. The vacuum term grows with time, while the other linear potentials $V_{C P^{-}}$and $V_{C P^{+}}^{i}$ decrease. The direction of an ANME configuration therefore rotates toward $\Delta$, the direction of vacuum-mass eigenstates. However, $\langle\mathbf{v}\rangle$ and $\left\langle\mathbf{w}^{*}\right\rangle$ remain pointing along the flavor axis $(1,0,0)$ because all the relevant time scales are initially below the $1 / H$ line. From Eqs. (12) 
and (18) one sees that eventually $\langle v\rangle$ begins to acquire a negative third component, while $\left\langle w^{*}\right\rangle$ acquires a positive one. The net result is a positive contribution to $B_{3}$ [cf. Eqs. (13)]. When

$$
\left(\left\langle v_{3}\right\rangle-\left\langle w_{3}^{*}\right\rangle\right) /(\langle|\mathbf{v}|\rangle+\langle|\mathbf{w}|\rangle)
$$

becomes

$$
\sim-\frac{1}{10} \frac{\tau_{v v}^{\min }}{1 / H},
$$

$\langle\mathbf{v}\rangle$ and $\left\langle\mathbf{w}^{*}\right\rangle$ begin to rotate toward $\Delta$. Thus, the nonlinear term is found to be responsible for the smooth precocious rotation to vacuum-mass eigenstates.

In Figs. 13 and 14 the linear and nonlinear cases are compared. As anticipated, in the linear case there is no rotation to vacuum-mass eigenstates until the $\left\langle\tau_{\text {vac }}\right\rangle$ lines crosses the $1 / H$ line, whereupon a "bounce" occurs. In contrast, the nonlinear case exhibits evolutionary behavior. Moreover, an examination of individual particle vectors reveals that particles with different energies rotate together, as expected since the nonlinear potential is energy independent.

In Fig. 15 we magnify the time near the crossover region and display the difference of ratios $r_{v 3}-r_{w^{*}}$. It is evident that a buildup of $\sim 10^{-7}$ in these quantities is sufficient to make $\tau_{v v}^{\min }$ a relevant time scale (cf. Fig. 2), and this is where $r_{v 1}$ and $r_{v 2}$ begin to change in Figs. 13 and 14.

Finally, we remark that in an area overlapping R3 and R4 near $\Delta=10^{-9} \mathrm{eV}^{2}$, both adiabaticity and the rotation mechanism play a role in evolutionary behavior. Figures 16 and 17 display the situation for $\sin ^{2} 2 \theta=0.81$ and 0.25 .

\section{B. Neutrino properties}

In this subsection, we discuss several properties of neutrino and antineutrino evolution that appear to be generic in the highly nonlinear regime, i.e., when $\left\langle\tau_{\mathrm{vac}}\right\rangle<<\tau_{v v}^{\min }$. We have studied these properties at many points of the $\sin ^{2} 2 \theta-\Delta$ plane. However, to save space we display re-

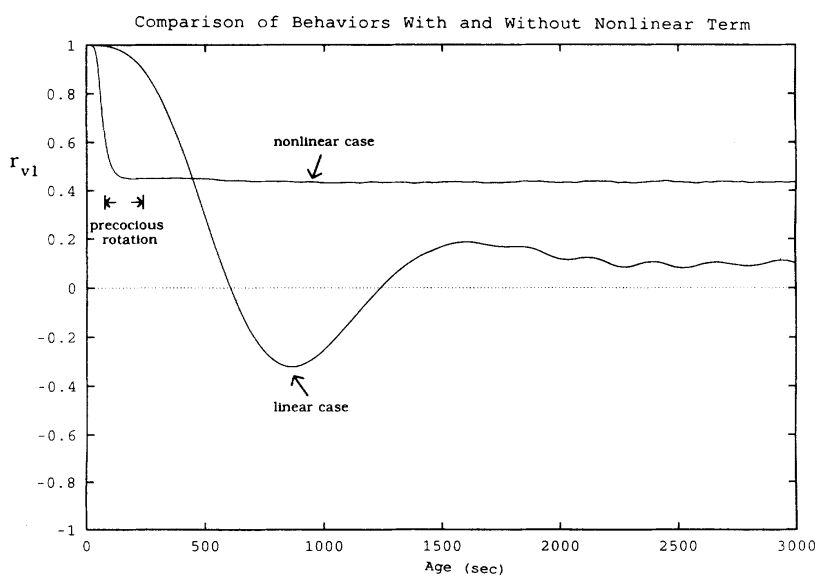

FIG. 13. Comparison of behaviors with and without the nonlinear term. The ratio $r_{v 1}$ is shown as a function of time for $\Delta=10^{-12} \mathrm{eV}^{2}$ and $\sin ^{2} 2 \theta=0.81$.

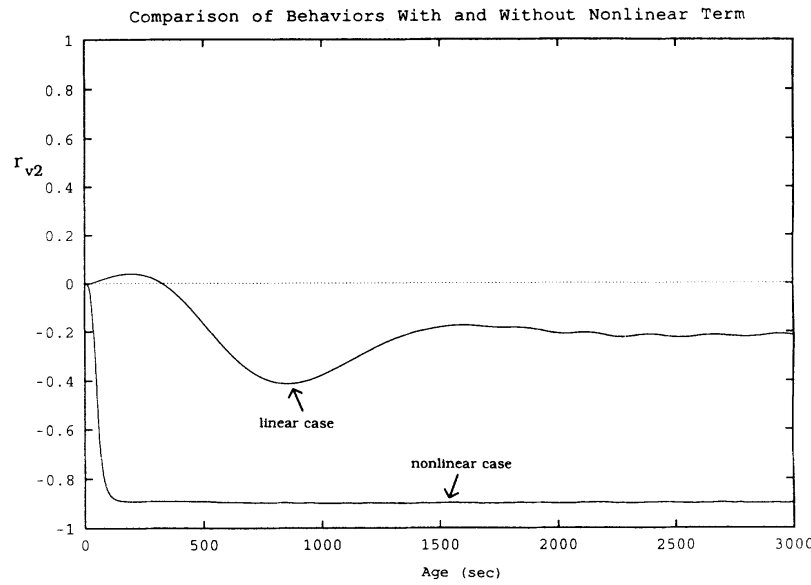

FIG. 14. Comparison of the linear and nonlinear cases. The ratio $r_{v 2}$ is shown as a function of time for $\Delta=10^{-12} \mathrm{eV}^{2}$ and $\sin ^{2} 2 \theta=0.81$.

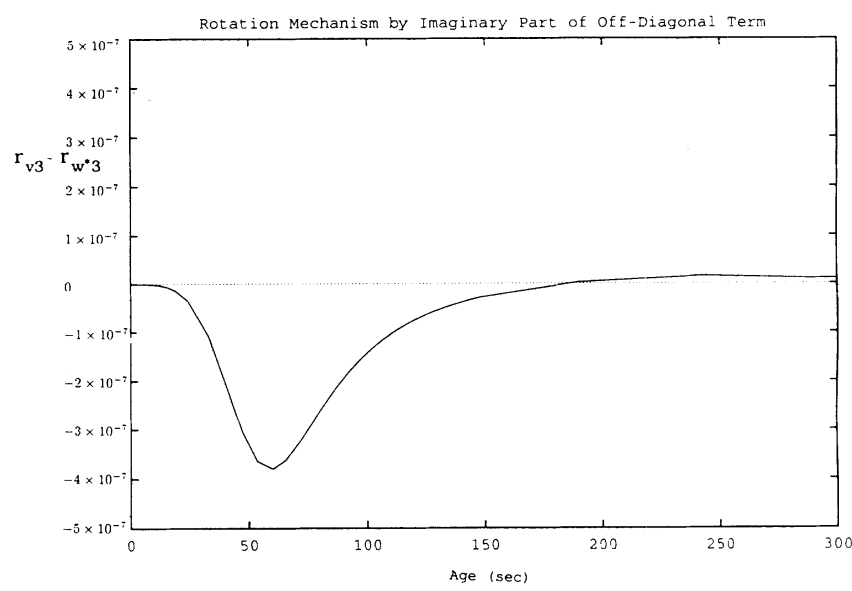

FIG. 15. The rotation mechanism. The difference of ratios $r_{v 3}-r_{w^{*} 3}$ is displayed as a function of time for $\Delta=10^{-12} \mathrm{eV}^{2}$ and $\sin ^{2} 2 \theta=0.81$. Note the magnification of the time scale relative to Fig. 14 .

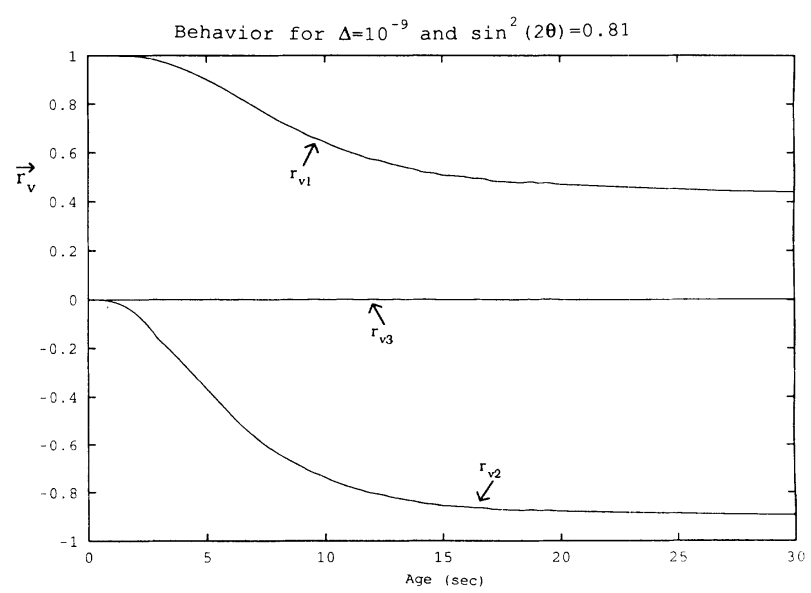

FIG. 16. Evolutionary neutrino behavior. The ratios $\mathbf{r}_{v}$ are displayed as a function of time for $\Delta=10^{-9} \mathrm{eV}^{2}$ and $\sin ^{2} 2 \theta=0.81$. 


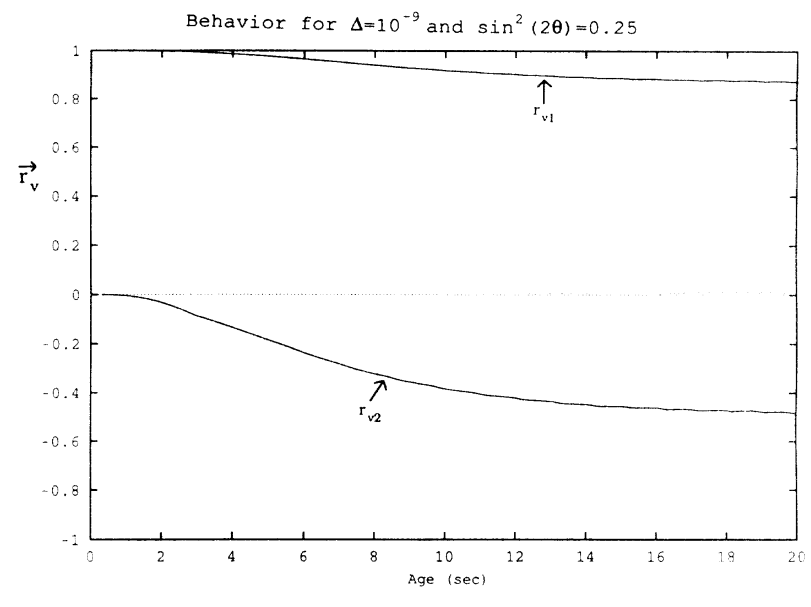

FIG. 17. The ratios $\mathbf{r}_{v}$ as a function of time for $\Delta=10^{-9} \mathrm{eV}^{2}$ and $\sin ^{2} 2 \theta=0.25$.

sults here only for the representative values $\Delta=10^{-4}$, $10^{-6}, 10^{-9}$, and $10^{-12} \mathrm{eV}^{2}$, and $\sin ^{2} 2 \theta=0.25$ and 0.81 . Also, in the tables that follow we consider three characteristic times during a run: an early point $a$; a point $b$ in the crossover region; and a point $c$ later in the run. The precise times are given in Table III for each of our representative points in the $\sin ^{2} 2 \theta-\Delta$ plane.

The first property we discuss is that particles maintain themselves in ANME configurations after being initialized therein. We call this the $A N M E$ property. The third column of Table IV displays the average of the test quantity

TABLE IV. Neutrino properties. This table examines some properties of neutrino behavior: the ANME property via $t_{\mathrm{ANME}}$, the related planar quantity via $t_{p}$, and the alignment property via $t_{a}$

\begin{tabular}{ccccc}
\hline \hline $\sin ^{2} 2 \theta$ & Point & $t_{\text {ANME }}$ & $t_{p}$ & $t_{a}$ \\
\hline 0.25 & $12 a$ & 0.999998 & 0.999999 & 1.000000 \\
& $12 b$ & 0.822638 & 0.999513 & 0.999891 \\
& $12 c$ & 0.999472 & 0.999752 & 0.999637 \\
& $9 a$ & 0.969034 & 0.999647 & 0.999974 \\
& $9 b$ & 0.944492 & 0.984594 & 0.994719 \\
& $9 c$ & 0.997797 & 0.997985 & 0.999466 \\
& $6 a$ & 0.999991 & 0.999996 & 0.996300 \\
& $6 b$ & 0.999989 & 0.999991 & 0.995408 \\
& $6 c$ & 0.999986 & 0.999997 & 0.999979 \\
& $4 a$ & 0.999998 & 1.000000 & 0.999984 \\
& $4 c$ & 0.977019 & 0.977285 & 0.999997 \\
& $12 a$ & 0.999995 & 0.999996 & 1.000000 \\
& $12 b$ & 0.663304 & 0.998458 & 0.999782 \\
& $12 c$ & 0.998649 & 0.999299 & 0.998652 \\
& $9 a$ & 0.999949 & 0.999971 & 0.999920 \\
& $9 b$ & 0.995491 & 0.999560 & 0.981697 \\
& $9 c$ & 0.994276 & 0.996034 & 0.996304 \\
& $6 a$ & 0.999955 & 0.999977 & 0.981655 \\
& $6 b$ & 0.999947 & 0.999976 & 0.981630 \\
& $6 c$ & 0.999990 & 0.999996 & 0.999955 \\
& $4 a$ & 0.999998 & 0.999998 & 0.999373 \\
& $4 c$ & 1.000000 & 1.000000 & 0.999970 \\
\hline \hline
\end{tabular}

$$
t_{\mathrm{ANME}}=\frac{1}{2}\left(\left\langle\left|\hat{\mathbf{v}} \cdot \widehat{\mathbf{B}}_{v}\right|\right\rangle+\left\langle\left|\hat{\mathbf{w}} \cdot \widehat{\mathbf{B}}_{w}\right|\right\rangle\right),
$$

where for a quantity $Q^{i}$ we define

$$
\langle Q\rangle=\frac{\sum_{i}\left|n_{v}^{i}\right| Q^{i}}{\sum_{i}\left|n_{v}^{i}\right|},
$$

where $n_{v}^{i}$ is given in Eq. (21). If $t_{\mathrm{ANME}}$ is close to one, the particles are in an ANME configuration. By construction, this is initially the case. Fluctuations in the nonlinear background term $\mathbf{V}_{v v}$ tend to cause $t_{\mathrm{ANME}}$ to move slowly away from one. Compare, for example, the value of $t_{\mathrm{ANME}}$ for $\sin ^{2} 2 \theta=0.25$ at points $4 a$ and $4 c$. The latter is reached after about 1000 oscillation times.

In $R 2$, the particles start in ANME configurations that are close to vacuum-mass eigenstates and remain close to vacuum-mass eigenstates, provided no parametric effects occur. In R3, particles start in ANME configurations that are near flavor eigenstates and rotate to ones that are approximate vacuum-mass eigenstates. As discussed in the previous subsection, this rotation is adiabatic. As a consequence, the particle vectors can maintain alignment with the effective magnetic fields. Hence, in R2 and R3 it is unsurprising that neutrinos maintain themselves in ANME configurations. We have explicitly checked that for $10^{-8} \mathrm{eV}^{2} \lesssim \Delta \lesssim 1 \mathrm{eV}^{2}$ the same property holds in the linear case, as is also to be expected.

If the nonlinear term fails to dominate, so the system is effectively linear, one might anticipate that nonadiabatic rotation would cause vectors to deviate from an ANME configuration and subsequently display other behavior, such as self-maintained coherence. It is instructive to set $\mathbf{V}_{v v}$ to zero. In the linear case at $\Delta=10^{-12} \mathrm{eV}^{2}$ and $\sin ^{2} 2 \theta=0.81, t_{\text {ANME }}$ is less than 0.5 at times after the crossover region. At $\Delta=10^{-9} \mathrm{eV}^{2}$ and $\sin ^{2} 2 \theta=0.81$, $t_{\mathrm{ANME}}=0.86$ beyond the crossover region, which is closer to one because the rotation is closer to being adiabatic. However, the nonlinear case displays robustness in this regard. Although for small $\Delta$ the quantity $t_{\mathrm{ANME}}$ can be far from unity in the crossover region (cf. the point $12 b$ for $\sin ^{2} 2 \theta=0.81$ ), it returns close to one at later times.

The reason for this is related to the rotation mechanism discussed in the previous subsection. A significant third component $B_{3}$ of the effective magnetic field arises in the crossover region due to the term $\left\langle v_{3}\right\rangle-\left\langle w_{3}^{*}\right\rangle$. This creates a mismatch between the particle vectors, which lie almost in the 1-2 plane. To verify this statement, we define a test based on the planar dot product:

$$
t_{p}=\frac{1}{2}\left(\left\langle\widehat{\mathbf{v}}_{p} \cdot \widehat{\mathbf{B}}_{v p}\right\rangle+\left\langle\hat{\mathbf{w}}_{p} \cdot \widehat{\mathbf{B}}_{w p}\right\rangle\right),
$$

where the subscript $p$ indicates that only the first two components of a vector are used. The fourth column in Table IV displays $t_{p}$. It is indeed close to one in the crossover region even for small $\Delta$. After passing through the crossover region, an ANME configuration reappears.

Most vectors tend to remain essentially in the 1-2 plane. We call this the planarity property. However, there are some exceptions. For example, we have ob- 
served low-energy neutrinos undergoing MSW conversion in runs with $\Delta \sim 10^{-9} \mathrm{eV}^{2}$. The values of $r_{v 3}$ and $r_{w 3}$ were no bigger than a few times $10^{-2}$ and usually orders of magnitude smaller.

The planarity property follows from the ANME property because an ANME configuration lies close to the 1-2 plane. In R4, the planarity property is to be expected since the behavior is adiabatic. In the linear case, planarity is also a property of the behavior for $10^{-8} \mathrm{eV}^{2} \lesssim \Delta$ because of adiabaticity. For smaller values of $\Delta$ in the linear case, the transient oscillatory behavior appearing near the crossover region produces sizable values of $r_{v 3}$ and $r_{w 3}$ for $\sin ^{2} 2 \theta$ sufficiently large. Eventually, decoherence sets in and $r_{v 3}$ and $r_{w 3}$ become small. However, the third components of individual vectors remain substantial. In contrast, in the full theory with the nonlinear term the average vector and most individual vectors lie almost in the 1-2 plane.

With the exception of low-energy MSW conversions, we have observed that individual vectors are also approximately aligned. We refer to this as the alignment property. This concept can be quantified in terms of the average dot product of the individual unit vectors with the unit average vector. We define the alignment test $t_{a}$ as

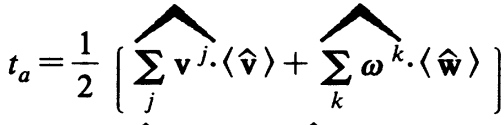

$$
\begin{aligned}
& \equiv \frac{1}{2}(\widehat{\langle v\rangle} \cdot\langle\hat{v}\rangle+\widehat{\langle w\rangle} \cdot\langle\hat{\mathbf{w}}\rangle) \text {. }
\end{aligned}
$$

The fifth column of Table IV presents values of $t_{a}$. These are close to one.

The alignment property can also be explained via the ANME property, since individual ANME configurations for different energy bins are for the most part aligned. This may be contrasted with the linear case. Although alignment holds for $10^{-9} \mathrm{eV}^{2} \lesssim \Delta$, for smaller values of $\Delta$ the vectors spread after passing through the crossover region. For example, for $\Delta=10^{-12} \mathrm{eV}^{2}$ and $\sin ^{2} 2 \theta=0.81$, $t_{a}$ becomes less than 0.5 .

Unlike the ANME property, alignment is also a feature of self-maintained coherence in the highly nonlinear regime. Moreover, alignment holds between the individual neutrino and antineutrino vectors $\mathbf{v}^{j}$ and $\mathbf{w}^{k}$. However, this is not true in the linear case in the crossover region and beyond for $10^{-9} \mathrm{eV}^{2} \lesssim \Delta$ and sizable mixing angles.

\section{C. $C P$ and the suppression mechanism}

In this subsection, we consider effects associated with the $C P$ asymmetry between the neutrino flavors. This is of interest in its own right as well as for physical issues such as nucleosynthesis or neutrino relic detection.

As a measure of $C P$ asymmetry, we define the three quantities

$$
\left.C P_{\alpha}^{\text {asy }}=\left|\left\langle v_{\alpha}\right\rangle-\left\langle w_{\alpha}\right\rangle\right| /(|\mathbf{v}|\rangle+\langle|\mathbf{w}|\rangle\right),
$$

where $\alpha=1,2,3$ labels the three-vector components. The quantity $C P_{1}^{\text {asy }}$ is proportional to the density difference

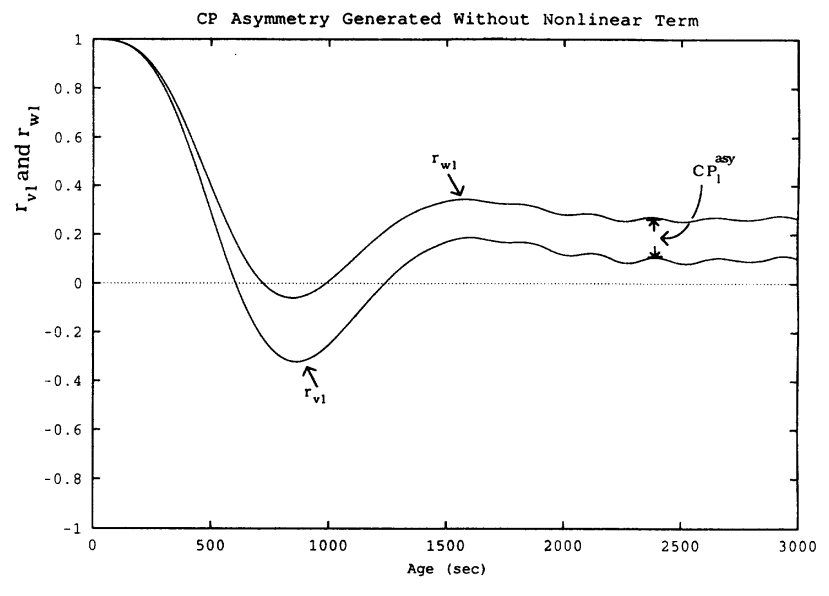

FIG. 18. $C P$ asymmetry in the linear case. The ratios $r_{v 1}$ and $r_{v 2}$ are shown as a function of time for $\Delta=10^{-12} \mathrm{eV}^{2}$ and $\sin ^{2} 2 \theta=0.81$.

between neutrinos and antineutrinos in the flavor basis. ${ }^{4}$ When mixing occurs, $C P_{2}^{\text {asy }}$ has a related physical interpretation since the neutrino-antineutrino density difference in the mass-eigenstate basis is proportional to a linear combination of $C P_{1}^{\text {asy }}$ and $C P_{2}^{\text {asy }}$. It is an open question as to how a sizable $C P_{3}^{\text {asy }}$ could directly affect experiment.

Relative to the linear case, we found that $C P$ asymmetry was suppressed in the full nonlinear system by several orders of magnitude. For the linear case, runs with $10^{-9} \mathrm{eV}^{2} \lesssim \Delta$ and $\sin ^{2} 2 \theta=0.81$ produced $C P$ asymmetry of the order of $10 \%$. This substantial accumulation arises throughout $\mathrm{R} 4$ because $V_{C P^{-}}$is larger than $V_{C P}^{i}$ in the crossover region. Figure 18 demonstrates that the $C P$ asymmetry is generated in the crossover region. For $\Delta=10^{-7}$ or $10^{-8} \mathrm{eV}^{2}$ and $\sin ^{2} 2 \theta=0.81$, the $C P$ asymmetry was of the order of $1 \%$ in the linear case, while for $\Delta \sim 10^{-6} \mathrm{eV}^{2}$ it was less than 0.0001 . For the nonlinear case, the biggest $C P$ asymmetry occurred in $C P_{3}^{\text {asy }}$. Although it became as big as a few percent in certain runs, it usually was much smaller. The smallness of $C P_{3}^{\text {asy }}$ is aided by the planarity property. In contrast, $C P_{1}^{\text {asy }}$ and $C P_{2}^{\text {asy }}$ were even smaller. Only for selected moments of a few runs did they approach $10^{-4}$. Usually they ranged from $10^{-5}$ to $10^{-7}$.

Given the ANME property, we can explain the $C P$ suppression in $C P_{1}^{\text {asy }}$ and $C P_{2}^{\text {asy }}$ as follows. Consider first the linear case. The biggest $C P$ asymmetry occurs when $\mathbf{B}_{-}=-V_{C P}-\widehat{\hat{e}}_{1}$ points in a direction perpendicular to the $C P$-conserving vector $\mathbf{B}_{+}=\Delta / 2 E-V_{C P}+\hat{e}_{1}$ of the other two neutrino potentials. This situation is illustrated schematically in Fig. 19(a), where the neutrino vectors are grouped into an average vector for simplicity. In the

\footnotetext{
${ }^{4}$ Since $C P_{1}^{\text {asy }}$ represents $C P$ asymmetry in the neutrinoantineutrino excess, the net $C P$ asymmetry is smaller by about 2 orders of magnitude.
} 
real case, $\mathbf{B}_{+}$is energy dependent and the directions of $\mathbf{B}_{v}=\mathbf{B}_{+}+\mathbf{B}_{-}$and its analogue $\mathbf{B}_{w^{*}}=-\mathbf{B}_{+}+\mathbf{B}_{-}$vary from bin to bin. Note also that $\sqrt{2} G_{F}\langle\mathbf{v}\rangle / a^{3}$ and $\sqrt{2} G_{F}\left\langle w^{*}\right\rangle / a^{3}$ are not drawn to scale: these vectors are about $10^{7}$ times longer than $\mathbf{B}_{-}$and at least $10^{6}$ times longer than $\mathbf{B}_{+}$. Their difference is the contribution to the background field. Assuming that the particles are in ANME configurations and using similar triangles, the figure shows that the $C P$ asymmetry should be of order $\left|\mathbf{B}_{-}\right| /\left|\mathbf{B}_{+}\right|$. This agrees in order of magnitude with the $C P$ asymmetry observed in our simulations of the linear case.

When the nonlinear term is also included, the vectors shown in Fig. 19(a) no longer form an ANME configuration because $\mathbf{B}_{v v} \equiv-\mathbf{V}_{v v}$ adds a significant contribution to $\mathbf{B}_{v}$ and $\mathbf{B}_{w^{*}}$. In this situation, an ANME configuration requires $\langle\mathbf{v}\rangle$ and $\left\langle\mathbf{w}^{*}\right\rangle$ to be approximately aligned. Figure 19(b) displays the configuration for the nonlinear case. Then, it follows that

$$
\frac{\left|\mathbf{B}_{-}\right|-\left|\mathbf{V}_{v v}\right|}{\left|\mathbf{B}_{-}\right|+\left|\mathbf{V}_{v v}\right|} \approx \frac{a^{3}\left|\mathbf{B}_{+}\right|}{2 \sqrt{2} G_{F}|\langle\mathbf{v}\rangle|} .
$$

Then, the vectors $\mathbf{B}_{v}=\mathbf{B}_{+}+\mathbf{B}_{-}+\mathbf{B}_{v v}$ and $\mathbf{B}_{w} *$ $=-\mathbf{B}_{+}+\mathbf{B}_{-}+\mathbf{B}_{v v}$ point almost along $\mathbf{B}_{+}$. These two vectors are not shown to avoid cluttering the figure. It can be seen that $C P_{1}^{\text {asy }}$ and $C P_{2}^{\text {asy }}$ are of order

$$
\frac{a^{3}\left|\mathbf{B}_{v v}\right|}{2 \sqrt{2} G_{F}|\langle\mathbf{v}\rangle|} \approx \frac{a^{3}\left|\mathbf{B}_{-}\right|}{2 \sqrt{2} G_{F}|\langle\mathbf{v}\rangle|} \approx 10^{-7} \text {. }
$$

Allowing for the fact that $C P$ suppression should be somewhat larger than this for ANME configurations, this agrees with what we observe numerically.

The above argument also holds if the $C P$ asymmetry is generated by only a few individual neutrino vectors. For example, in runs with $\Delta=10^{-9} \mathrm{eV}^{2}$ low-energy neutrinos are MSW rotated whereas antineutrinos remain unaffected. The fraction of neutrinos with low-enough energy for conversion is $3 \times 10^{-4}$, so the MSW rotation
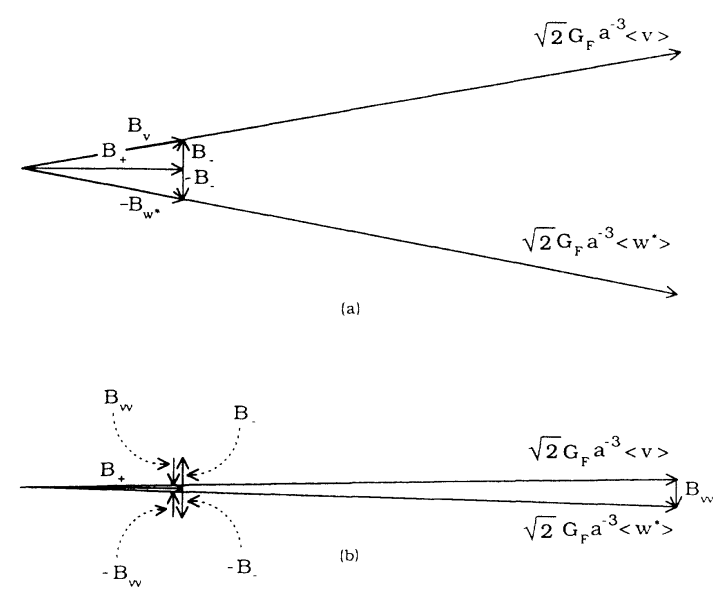

FIG. 19. (a) Vector diagram for estimating $C P$ asymmetry in the linear case. (b) Vector diagram for estimating $C P$ asymmetry in the nonlinear case, under the assumption that particles are in ANME configurations. generates a $\mathbf{B}_{-}$no larger than a factor of $3 \times 10^{-4}$ of $2 \sqrt{2} G_{F}|\langle\mathbf{v}\rangle| / a^{3}$. However, the neutrinos and antineutrinos vectors react to maintain ANME configurations and so the observed $C P$ asymmetry is much smaller.

This $C P$ suppression mechanism does not directly apply to $C P_{3}^{\text {asy }}$. However, it does apply to a similar-seeming but different quantity

$$
\left|\left\langle v_{3}\right\rangle-\left\langle w_{3}^{*}\right\rangle\right| /(\langle|\mathbf{v}|\rangle+\langle|\mathbf{w}|\rangle) \text {. }
$$

Simulations show this quantity is indeed suppressed relative to $C P_{3}^{\text {asy }}$. This provides additional support for our explanation of the $C P$ suppression. Moreover, our argument also provides a reason why the background ratio

$$
\left|\langle\mathbf{v}\rangle-\left\langle\mathbf{w}^{*}\right\rangle\right| /(\langle|\mathbf{v}|\rangle+\langle|\mathbf{w}|\rangle)
$$

tends to be small, usually ranging from $10^{-5}$ to $10^{-7}$ except at times before the crossover region where it is even smaller.

The reader should note that a larger $C P$ asymmetry might arise from parametric effects. Although this does not appear in any of our present data, including those over thousands of oscillation times, a relatively large $C P$ asymmetry could, in principle, accumulate over much longer time scales.

The $C P$ asymmetry we have seen is several orders of magnitude smaller than that reported in Ref. [9] for $\Delta \sim 10^{-7} \mathrm{eV}^{2}$ where two dominant effects, $V_{C P^{+}}^{i}$ and $\mathbf{V}_{v v}$, were disregarded. Our results suggest that in the early Universe a very small $C P$ asymmetry is likely to be maintained through much of the critical period when neutrinos could affect nucleosynthesis. If true, $C P$-asymmetry effects are minimal. The major effect arises from the flavor-excess distortions, implying a shift in the ${ }^{4} \mathrm{He}$ abundance of $\Delta Y \sim(1-2) \times 10^{-4}[2,3,9]$.

Another consequence of the $C P$ suppression is an increase in the difficulty of detecting the relic neutrino background. Assuming no unexpected parametric effects, techniques dependent on forward scattering [22] may be impractical.

\section{Approximation scheme for particle vectors}

Combining the ANME property with the argument for $C P$ suppression in the previous subsection demonstrates that, to a good approximation, the component of $\mathbf{B}_{-}$that is not aligned along $\mathbf{B}_{+}$cancels with the nonlinear term $\mathbf{V}_{v v}$. This suggests that mass eigenstates determined solely from $V_{C P^{+}}^{i}$ and $\Delta / 2 E^{i}$, i.e., $\mathbf{B}_{+}$in Fig. 19 , should be ANME configurations. It then follows that $\mathbf{v}^{j}$ and $\mathbf{w}^{* k}$ should be well approximated by calculations involving only these terms.

The energy dependence in $V_{C P}^{j}+$ implies the existence of a different $\mathbf{B}_{+}^{j}$ for each neutrino vector. The same holds for the analogous formulas for antineutrinos. The evolutionary behavior implies $\mathbf{v}^{j}$ and $\mathbf{w}^{* k}$ should be rotated onto $\mathbf{B}_{+}^{i}$ rather than projected. The only ambiguity is whether the $\mathbf{v}^{j}$ and $\mathbf{w}^{* k}$ point along or against $\widehat{\mathbf{B}}_{+}^{i}$. The natural choice is to minimize the vector rotation. With this approach, we obtain an analytical approximation given by 
TABLE V. Comparison of approximation with simulation: average vectors.

\begin{tabular}{cccccc}
\hline \hline $\sin ^{2} 2 \theta$ & Point & $v_{1}($ simul.) & $v_{1}$ (approx.) & $v_{2}$ (simul.) & $v_{2}$ (approx.) \\
\hline 0.25 & $12 a$ & 0.99853 & 0.99853 & -0.00050 & -0.00056 \\
& $12 b$ & 0.97510 & 0.96978 & -0.21464 & -0.21851 \\
& $12 c$ & 0.86900 & 0.86496 & -0.49119 & -0.49891 \\
& $9 a$ & 0.99852 & 0.99853 & -0.00446 & -0.00428 \\
& $9 b$ & 0.96515 & 0.96558 & -0.23662 & -0.23597 \\
& $9 c$ & 0.87381 & 0.87512 & -0.48280 & -0.48058 \\
& $6 a$ & 0.98198 & 0.98198 & -0.15993 & -0.15993 \\
& $6 b$ & 0.96516 & 0.96515 & -0.23767 & -0.23768 \\
& $6 c$ & 0.86800 & 0.86789 & -0.49358 & -0.49376 \\
& $4 a$ & 0.86800 & 0.86804 & -0.49356 & -0.49350 \\
& $4 c$ & 0.86650 & 0.86595 & -0.49619 & -0.49718 \\
& $12 a$ & 0.99853 & 0.99853 & -0.00092 & -0.00100 \\
& $12 b$ & 0.96749 & 0.94393 & -0.24634 & -0.27535 \\
& $12 c$ & 0.43390 & 0.43525 & -0.89804 & -0.89868 \\
$9 a$ & 0.99849 & 0.99854 & -0.00810 & -0.00774 \\
& $9 b$ & 0.94508 & 0.94829 & -0.26278 & -0.26386 \\
& $9 c$ & 0.46975 & 0.47046 & -0.87767 & -0.88063 \\
& $6 a$ & 0.92668 & 0.92668 & -0.32062 & -0.32062 \\
& $6 b$ & 0.71405 & 0.71410 & -0.67158 & -0.67169 \\
$6 c$ & 0.44378 & 0.44410 & -0.89446 & -0.89429 \\
& $4 a$ & 0.47377 & 0.47377 & -0.87827 & -0.87827 \\
$4 c$ & 0.44340 & 0.44340 & -0.89465 & -0.89465 \\
\hline \hline
\end{tabular}

$$
\mathbf{v}^{i}=\mathbf{w}^{* i}= \pm N_{v}^{i} \hat{\mathbf{B}}_{+}^{i} .
$$

Our approximation gives zero $C P_{\alpha}^{\text {asy }}$ and zero third components $v_{3}^{j}$ and $w_{3}^{* k}$. It thereby implements extreme versions of the planarity and $C P$-suppression properties. Table $\mathrm{V}$ presents a comparison of results from the full simulation with numerical values given by the approximation. The first two components of $\langle\mathbf{v}\rangle$ are shown at the canonical points in the $\sin ^{2} 2 \theta-\Delta$ plane. Overall, there is agreement to roughly three decimal places. The biggest difference occurs in the crossover region for small $\Delta$; cf. point $12 b$, where about two decimal places are reproduced.

TABLE VI. Comparison of approximation with simulation: individual neutrino vectors.

\begin{tabular}{rrrrrr}
\hline \hline Bin & $v_{1}$ (simul.) & $v_{1}$ (approx.) & $v_{2}$ (simul.) & $v_{2}$ (approx.) & $v_{3}$ (simul.) \\
\hline 2 & 0.00004 & -0.00002 & -0.00003 & 0.00005 & 0.00002 \\
4 & 0.00004 & -0.00009 & -0.00015 & 0.00018 & -0.00012 \\
6 & 0.00025 & -0.00021 & -0.00025 & 0.00042 & -0.00031 \\
8 & 0.00566 & -0.00356 & -0.00124 & 0.00701 & -0.00531 \\
10 & -0.00402 & -0.00356 & 0.00445 & 0.00537 & -0.00236 \\
12 & 0.76407 & 0.81068 & -0.72032 & -0.68234 & -0.14192 \\
14 & 1.76585 & 1.77635 & -1.05957 & -1.05810 & -0.18468 \\
16 & 1.26334 & 1.26270 & -0.56539 & -0.57024 & -0.06249 \\
18 & 1.41271 & 1.41115 & -0.54427 & -0.54954 & -0.03696 \\
20 & 1.20144 & 1.20009 & -0.40485 & -0.40900 & -0.01078 \\
22 & 0.61421 & 0.61352 & -0.18808 & -0.19034 & 0.00123 \\
24 & 1.23492 & 1.23362 & -0.35403 & -0.35865 & 0.00949 \\
26 & 1.53103 & 1.52981 & -0.39430 & -0.39983 & 0.02559 \\
28 & 1.48061 & 1.47963 & -0.33700 & -0.34318 & 0.03610 \\
30 & 1.40033 & 1.39961 & -0.28322 & -0.28979 & 0.04208 \\
32 & 2.54357 & 2.54254 & -0.44627 & -0.46057 & 0.08781 \\
34 & 2.07507 & 2.07446 & -0.29351 & -0.30794 & 0.07839 \\
36 & 1.61081 & 1.61048 & -0.18681 & -0.19921 & 0.06107 \\
38 & 1.20172 & 1.20158 & -0.11641 & -0.12564 & 0.04371 \\
40 & 0.86780 & 0.86778 & -0.07162 & -0.07766 & 0.02959 \\
42 & 0.92549 & 0.92562 & -0.06076 & -0.06489 & 0.02772 \\
44 & 0.68870 & 0.68895 & -0.03618 & -0.03589 & 0.01783 \\
46 & 0.04395 & 0.04398 & -0.00162 & -0.00126 & 0.00117 \\
48 & 0.00282 & 0.00282 & -0.00006 & -0.00005 & 0.00008 \\
50 & 0.00004 & 0.00004 & 0.0 & 0.0 & 0.0 \\
\hline \hline
\end{tabular}


TABLE VII. Comparisons of approximation with simulation: individual antineutrino vectors.

\begin{tabular}{rrrrrr}
\hline \hline Bin & $v_{1}$ (simul.) & $v_{1}$ (approx.) & $v_{2}$ (simul.) & $v_{2}$ (approx.) & \multicolumn{1}{c}{$v_{3}$ (simul.) } \\
\hline 2 & -0.00003 & -0.00002 & 0.00004 & 0.00005 & -0.00002 \\
4 & -0.00008 & -0.00009 & 0.00018 & 0.00018 & 0.00003 \\
6 & -0.00020 & -0.00021 & 0.00042 & 0.00042 & 0.00008 \\
8 & -0.00257 & -0.00356 & 0.00738 & 0.00701 & 0.00084 \\
10 & -0.00198 & -0.00356 & 0.00606 & 0.00537 & 0.00097 \\
12 & 0.76430 & 0.81068 & -0.72053 & -0.68234 & 0.13961 \\
14 & 1.76719 & 1.77635 & -1.06132 & -1.05810 & 0.16019 \\
16 & 1.26570 & 1.26270 & -0.56145 & -0.57024 & 0.04875 \\
18 & 1.41516 & 1.41115 & -0.53854 & -0.54954 & 0.02551 \\
20 & 1.20311 & 1.20009 & -0.39999 & -0.40900 & 0.00389 \\
22 & 0.61485 & 0.61352 & -0.18596 & -0.19034 & -0.00323 \\
24 & 1.23595 & 1.23362 & -0.35031 & -0.35865 & -0.01270 \\
26 & 1.53203 & 1.52981 & -0.39033 & -0.39983 & -0.02645 \\
28 & 1.48111 & 1.47963 & -0.33495 & -0.34318 & -0.03452 \\
30 & 1.40051 & 1.39961 & -0.28278 & -0.28979 & -0.03865 \\
32 & 2.54331 & 2.54254 & -0.44942 & -0.46057 & -0.07902 \\
34 & 2.07434 & 2.07446 & -0.30077 & -0.30794 & -0.06992 \\
36 & 1.61003 & 1.61048 & -0.19524 & -0.19921 & -0.05493 \\
38 & 1.20107 & 1.20158 & -0.12410 & -0.12564 & -0.04004 \\
40 & 0.86733 & 0.86778 & -0.07776 & -0.07766 & -0.02778 \\
42 & 0.92502 & 0.92562 & -0.06757 & -0.06489 & -0.02745 \\
44 & 0.68843 & 0.68895 & -0.04052 & -0.03589 & -0.01901 \\
46 & 0.04394 & 0.04398 & -0.00174 & -0.00126 & -0.00128 \\
48 & 0.00282 & 0.00282 & -0.00006 & -0.00005 & -0.00009 \\
50 & 0.00004 & 0.00004 & 0.0 & 0.0 & 0.0 \\
\hline \hline
\end{tabular}

The approximation works best for large $\Delta$ at early times, away from the crossover region. Tables VI and VII contain the comparison for individual neutrino and antineutrino vectors, respectively, for $\sin ^{2} 2 \theta=0.81$ at the point $9 b$. To save space, only every other bin is shown for a 50-bin division of the energy spectrum. Also, we have not shown the approximation for $v_{3}^{j}$ or $w_{3}^{* k}$ since they are always zero. The true numbers are $10^{-4}$ times the ones displayed. In most bins there is three-figure agreement. The approximation is better for higherenergy bins because $V_{C P}^{i}+$ is relatively more important than $V_{C P}$ - there. This should be contrasted with the lowest-energy vectors, for which even the sign is incorrect. Point $9 b$ was specially selected to illustrate this effect. The low-energy neutrinos have undergone MSW conversion and hence are pointing approximately in the opposite direction to the others. Note that the corresponding low-energy antineutrinos have not undergone MSW conversion and so there is better agreement.

\section{CONCLUSION}

In this paper, we have derived equations governing neutrino and antineutrino behaviors in the early Universe and have presented results from numerical simulations. The analysis incorporates the nonlinear effects of the neutrino-antineutrino background as well as leading $C P$ symmetric and $C P$ asymmetric terms arising from the interactions with electrons and positrons.
The observed behaviors of neutrinos and antineutrinos depend on the values of $\sin ^{2} 2 \theta$ and $\Delta$. The behavior ranges from vacuumlike at relatively large values of $\Delta$ to evolutionary or to oscillations exhibiting self-maintained coherence. Several neutrino properties arising from the nonlinear neutrino self-interactions can be identified, including maintenance in ANME configurations, planarity, and alignment. The nonlinear effects tend to suppress any $C P$ asymmetry relative to the linear case. We have also presented an analytical approximation scheme for particle densities.

In the absence of possible parametric effects such as self-maintained coherence, the $C P$ suppression mechanism suggests that the effect of the flavor excess on nucleosynthesis is minimal. It also implies that the cosmicneutrino relic may be more difficult to detect than expected. In contrast, if self-maintained coherence is realized in nature one can speculate about more striking effects, including consequences for dark matter and for the largescale structure of the Universe.

\section{ACKNOWLEDGMENTS}

We thank Jim Pantaleone for numerous discussions and for collaboration during the early stages of this project. We also thank Archie Hendry for useful conversations. Some of this research was performed while V.A.K. was visiting the Aspen Center for Physics. This work was supported in part by the United States Department of Energy under Grant Nos. DE-FG02-91ER40661 and DE-FG02-92ER40698. 
[1] See, for example, E. W. Kolb and M. S. Turner, The Early Universe (Addison Wesley, Redwood City, CA, 1990).

[2] S. Dodelson and M. S. Turner, Phys. Rev. D 46, 3372 (1992); B. Fields, S. Dodelson, and M. S. Turner ibid. 47, 4309 (1993).

[3] A. D. Dolgov and M. Fukugita, Phys. Rev. D 46, 5378 (1992).

[4] T. P. Walker, G. Steigman, D. N. Schramm, K. A. Olive, and H. S. Kang, Astrophys. J. 376, 51 (1991).

[5] K. A. Olive, D. N. Schramm, D. Thomas, and T. P. Walker, Phys. Lett. B 265, 239 (1991).

[6] For reviews, see, for example, P. F. Smith, in Trends in Astroparticle Physics, edited by D. B. Cline and R. Peccei (World Scientific, Singapore, 1990); P. F. Smith and J. D. Lewin, Phys. Rep. 187, 203 (1990).

[7] For a review, see, for example, T. K. Kuo and J. Pantaleone, Rev. Mod. Phys. 61, 937 (1989).

[8] A. D. Dolgov, Yad. Fiz. 33, 1309 (1981) [Sov. J. Nucl. Phys. 33, 700 (1981)].

[9] P. Langacker, S. T. Petcov, G. Steigman, and S. Toshev, Nucl. Phys. B282, 589 (1987).

[10] M. J. Savage, R. A. Malaney, and G. M. Fuller, Astrophys. J. 368, 1 (1991).
[11] K. Enqvist, K. Kainulainen, and J. Maalampi, Nucl. Phys. B349, 754 (1991).

[12] X. Shi, D. N. Schramm, and B. D. Fields, University of Chicago Report, 1993 (unpublished).

[13] L. Stodolsky, Phys. Rev. D 36, 2273 (1987).

[14] M. J. Thomson and B. McKellar, Phys. Lett. B 259, 113 (1991).

[15] J. Pantaleone, Phys. Lett. B 287, 128 (1992).

[16] J. Pantaleone, Phys. Rev. D 46, 510 (1992).

[17] S. Samuel, Phys. Rev. D 48, 1462 (1993).

[18] V. A. Kostelecký, J. Pantaleone, and S. Samuel, Phys. Lett. B 315, 46 (1993).

[19] G. Sigl and G. Raffelt, Max-Planck Institute Report No. MPI-Ph/92-112 (unpublished).

[20] D. Notzold and G. Raffelt, Nucl. Phys. B307, 924 (1988).

[21] L. Wolfenstein, Phys. Rev. D 17, 2369 (1978); 20, 2634 (1979); S. P. Mikheyev and A. Yu. Smirnov, Yad. Fiz. 42, 1441 (1985) [Sov. J. Nucl. Phys. 42, 913 (1985)]; Usp. Fiz. Nauk 153, 3 (1987) [Sov. Phys. Usp. 30, 759 (1987)].

[22] R. Opher, Astron. Astrophys. 37, 135 (1974); L. Stodolsky, Phys. Rev. Lett. 34, 110 (1975); R. R. Lewis, Phys. Rev. D 21, 663 (1980). 


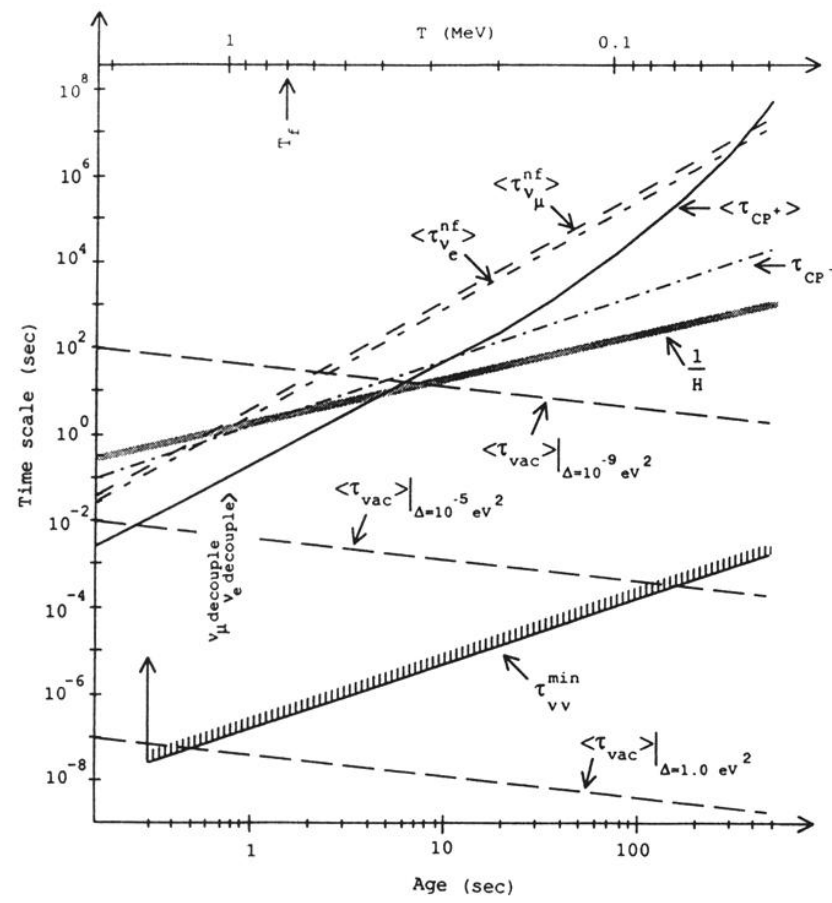

FIG. 2. Relevant time scales. Key time scales are displayed as a function of the age $t$ of the Universe. The time scale $\left\langle\tau_{\text {vac }}\right\rangle$ is displayed for the cases $\Delta=10^{-9}, 10^{-5}$, and $1 \mathrm{eV}^{2}$. For each factor of 10 increase (or decrease) in $\Delta$, the $\left\langle\tau_{\text {vac }}\right\rangle$ line moves down (or up) by one power of ten. The $1 / H$ line is drawn with width as a reminder that it acts as an order-of-magnitude estimate of the time at which a scale becomes insignificant. After the temperature of the Universe has dropped below $T_{f}$, neutrino flavor effects can no longer influence nucleosynthesis. The average nonforward scattering time scales are plotted separately for $v_{e}$ and $v_{\mu}$ for an average energy of $\bar{E}=3.151 T$. The averages $\left\langle\tau_{C P}{ }^{+}\right\rangle$and $\left\langle\tau_{\text {vac }}\right\rangle$ are graphed for an average energy $\bar{E} \approx 5 T$. The arrow on the $\tau_{v v}^{\min }$ line indicates that this quantity becomes effectively infinite at times $t<0.328 \mathrm{~s}$, as a result of our stepfunction approximation for the initial neutrino profile. The hatches on the $\tau_{v v}^{\min }$ line are a reminder that it provides a lower bound for $\tau_{v v}$. 


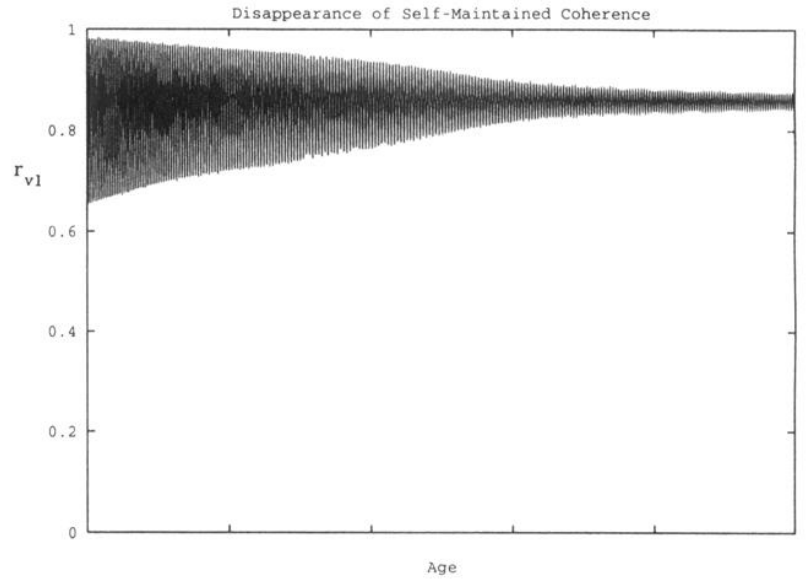

FIG. 7. Disappearance of self-maintained coherence. The small amplitude eventually shrinks to zero. 